\begin{tabular}{llll}
\hline Submission: 20/Feb/2021; & $1^{\text {st }}$ round notif.: 05/May/2021; & New version: 24/May/2021; & $2^{\text {nd }}$ round notif.: 29/Jun/2021; \\
Camera ready: 12/Jul/2021; & Edition review: 25/Jul/2021; & Available online: 06/Aug/2021; Published: 06/Aug/2021;
\end{tabular}

\title{
Revisión sistemática de la literatura sobre los sistemas tutores afectivos: 2001-2020
}

Title: A systematic review of the literature on affective tutoring systems: 2001-2020

\author{
Arlem Aleida Castillo Avila \\ Benemérita Universidad Autónoma de Puebla \\ arlemaleida.castilloavila@viep.com.mx
}

Josefina Guerrero García

Benemérita Universidad Autónoma de Puebla

josefina.guerrero@correo.buap.mx

\author{
Juan Manuel Gonzalez Calleros \\ Benemérita Universidad Autónoma de Puebla \\ juanmanuel.gonzalez@correo.buap.mx
}

\begin{abstract}
Resumen
El interés en el desarrollo de sistemas tutores inteligentes ha generado una amplia gama de estudios de investigación multidisciplinaria, así como el desarrollo de herramientas para distintas aplicaciones. Entre ellos se encuentran aquellos tutores que integran el estado afectivo del estudiante cuando tiene una sesión interactiva. Se realizó una revisión sistemática de la literatura en torno a los sistemas tutores inteligentes que toman en cuenta las emociones, denominados sistemas tutores afectivos, entre el periodo de 2001 y 2020. Este documento reporta los resultados de un análisis bibliométrico a un conjunto de 198 documentos obtenidos de la Web of Science, Scopus, ERIC y Dimensions. Se reportan los principales hallazgos con relación a 7 preguntas de investigación, de las cuales 3 implicaron un análisis cualitativo, finalmente se dan algunas conclusiones preliminares tomando en cuenta el escenario de México con respecto al desarrollo de los sistemas tutores afectivos.
\end{abstract}

\begin{abstract}
The interest in the development of intelligent tutoring systems has generated a wide range of multidisciplinary research studies, as well as the development of tools for different applications. Among them are those tutors that integrate the affective state of the student when having an interactive session. A systematic review of the literature on emotion-aware intelligent tutoring systems, called affective tutoring systems, was conducted between 2001 and 2020. This paper reports the results of a bibliometric analysis to a set of 198 papers obtained from the Web of Science, Scopus, ERIC and Dimensions. The main findings are reported in relation to 7 research questions, 3 of which involved a qualitative analysis, and finally some preliminary conclusions are given, considering the situation of Mexico regarding the development of affective tutoring systems.
\end{abstract}

Keywords: Affective Tutoring Systems, Intelligent Tutoring Systems, Computer-Mediated Learning.

Cite as: Castillo Avila, A. A., González Calleros, J. M., \& Guerrero García, J. (2021). Revisión sistemática de la literatura sobre los sistemas tutores afectivos: 2001-2020. Revista Brasileira de Informática na Educação, 29, 928-956. DOI: 10.5753/RBIE.2021.29.0.928 


\section{Introducción}

Dentro del proceso de aprendizaje influyen factores más allá del contexto cognitivo del estudiante, como lo son su personalidad, preferencias y estados afectivos. Para la investigación educativa ha sido de interés analizar y evaluar el aprendizaje y el desempeño del estudiante desde diferentes perspectivas. Se han evaluado las motivaciones, intereses, nivel de confianza, entre otras variables, para identificar el impacto y la relación que tienen con la cognición y el objetivo general del proceso de aprendizaje. En la modalidad de educación presencial, el profesor influye en el estado emocional inmediato de los estudiantes y esto a su vez en la efectividad del proceso educativo (Mohanan, Stringfellow, y Gupta, 2017). Sin embargo, cada persona experimenta una reacción diferente a una misma situación; por lo que la toma de decisiones con respecto a las actividades pedagógicas, contenido temático, y uso de recursos educativos, tendrá efectos distintos para un mismo grupo de estudiantes. Diversos investigadores dentro del campo de la enseñanza y las ciencias cognitivas han puntualizado en la necesidad de la personalización del proceso de enseñanza como un punto clave para optimizar el aprendizaje y lograr que sea significativo para el estudiante (Erümit y Çetin, 2020; VanLehn, 2011). Incluso dentro del tratamiento de los distintos trastornos específicos del aprendizaje, una de las estrategias en común es la personalización del "aula", refiriéndose al escenario en donde se lleve a cabo la sesión de enseñanza.

Dentro del conjunto de las tecnologías del aprendizaje y conocimiento (TAC), existen herramientas que permiten la mediación, personalización y evaluación continua del aprendizaje, como son los sistemas tutores inteligentes (ITS, por sus siglas en inglés). Estos sistemas se componen de cuatro modelos que incluyen las principales entidades y actores presentes en el proceso de aprendizaje (Cruces y De Arriaga, 2000). Han sido utilizados como complemento al aprendizaje presencial; es decir, bajo el paradigma del aprendizaje combinado (b-learning), y también como implementaciones del proceso de educación a distancia, electrónico y con uso de dispositivos móviles (e-learning y m-learning), sin la intervención directa del docente. Debido a su arquitectura basada en modelos se pueden diseñar ITS donde se incluyan variables necesarias para una entidad en particular; por ejemplo, el estado emocional del estudiante. Así mismo, existen los ITS adaptativos que modifican parte de su comportamiento ante los cambios en sus componentes para lograr un objetivo, en este caso, mejorar la efectividad del proceso de aprendizaje a través de la adaptación del material educativo y el ajuste a las instrucciones utilizadas durante la sesión interactiva con el sistema.

El concepto de ITS surge en la década de los 70's y desde entonces ha habido una amplia gama de investigaciones alrededor del mismo. La naturaleza divergente del proceso de aprendizaje y sus aplicaciones en diferentes campos, así como las disciplinas que envuelven a los sistemas tutores, han generado varias ramas de investigación, enfoques, técnicas y usos de los ITS, por lo que existe una gran cantidad de estudios que enfocan el tema desde diferentes perspectivas e incluso desde diferentes áreas del conocimiento. En esta investigación se tiene interés particular en el desarrollo de los procesos de aprendizaje mediado por tecnología, por lo que el enfoque es exclusivo para los tutores académicos.

Se realizó una revisión sistemática de la literatura más relevante sobre los ITS que toman en cuenta la integración de las emociones en el proceso de enseñanza-aprendizaje o en la evaluación final. Recientemente, Mousavinasab y colaboradores, han realizado una revisión sistemática de las características, aplicaciones y métodos de evaluación de los ITS, del periodo 2007 - 2017 (Mousavinasab y cols., 2021). Además de la cobertura temporal, los autores de (Mousavinasab y cols., 2021) hacen énfasis en las técnicas de inteligencia artificial aplicadas en el desarrollo de ITS, a diferencia del presente trabajo que se enfoca en la integración del estado afectivo en el proceso de aprendizaje dentro de los ITS, cuyas características no son reportadas en el trabajo de Mousavinasab y colaboradores. 
En el resto del documento se reportan los hallazgos estadísticos sobre los resultados de la primera etapa de la revisión sistemática. Así como un análisis cualitativo de una selección de los documentos más relevantes que permite identificar los retos y oportunidades en la investigación en torno a los sistemas tutores afectivos.

\section{Método}

Se realizó una búsqueda de los estudios de investigación en torno a la educación mediada por sistemas tutores inteligentes que integran el estado afectivo del estudiante, además del contexto cognitivo, dentro del modelo y proceso de aprendizaje que utilizan en las sesiones interactivas. La metodología de revisión está basada en la seguida por Xie et al. en (Xie, Chu, Hwang, y Wang, 2019) y las recomendaciones de Okoli en (Okoli y Schabram, 2010). El criterio de búsqueda surge a partir de la definición de 3 descriptores principales: learning outcomes, intelligent tutoring systems, y emotions. Las bases de datos utilizadas son Scopus, Web of Science, ERIC y Dimensions. La consulta ingresada en los sistemas de búsqueda varía por el lenguaje utilizado en cada plataforma, pero corresponde a una misma expresión lógica, ("learning outcome" AND ("intelligent tutoring system" OR "affective tutoring system" OR "intelligent tutor" OR "cognitive tutor") AND (emotion OR emotions OR affection OR "affective state”)).

En la primera etapa de la revisión de la literatura se realizó un análisis bibliométrico en el que a partir de información estadística se da respuesta a las siguientes preguntas de investigación: (1) ¿Cuáles son las principales fuentes de publicación con relación a los ITS que integran las emociones en el proceso de aprendizaje? (2) ¿Quiénes son los principales autores en relación con el problema específico de los tutores inteligentes afectivos? (3) ¿Cuáles son los conceptos más relevantes con relación a la evaluación del aprendizaje mediado por un ITS? (4) ¿Cuáles son las principales disciplinas en las que se ha hecho investigación sobre los ITS adaptativos a las emociones?

Después de realizar el análisis bibliométrico, se realizó un filtrado de los documentos tomando en cuenta 4 criterios de exclusión, (a) aquellos estudios que no se refieren a tutores como herramientas tecnológicas, (b) aquellos estudios cuya contribución no contempla el resultado en el aprendizaje de forma objetiva, (c) aquellos que se refieren a agentes afectivos en lugar de tutores afectivos, es decir, sistemas que no están compuestos de los cuatro modelos característicos de un ITS (Cruces y De Arriaga, 2000) y (d) libros de fundamentos teóricos sin aportación al estado del arte. Con el conjunto resultado de este proceso de filtrado se llevó a cabo un análisis cualitativo para responder a las siguientes preguntas de investigación, (5) ¿Cuáles son los hallazgos más relevantes en la integración de las emociones dentro de los sistemas tutores inteligentes?, (6) ¿Cuál es el efecto en el proceso de aprendizaje del uso de los sistemas tutores afectivos?, (7) ¿Cuál es el estado de la investigación de los sistemas tutores afectivos en México?

\subsection{Clasificación y análisis bibliométrico}

Los documentos se clasificaron a partir de cinco características relevantes con respecto al impacto y actualidad de las investigaciones, incluyendo (a) Años, (b) Fuentes de publicación, (c) Autores, (d) Tipos de publicaciones, y (e) Áreas de conocimiento. El objetivo del análisis de la primera etapa es examinar las tendencias y patrones de los documentos en relación con las características mencionadas. 


\section{Resultados}

En la primera etapa de la revisión sistemática se obtuvieron un total de 195 documentos, publicados entre el 2001 hasta el 2020, provenientes de las bases de datos Scopus, Web of Science, ERIC y Dimensions. La tendencia de producción en este periodo se muestra en la Figura 1. Se observa una tendencia al alza que tiene puntos altos en 2010, 2013 y 2019. La Figura 2 muestra la distribución de los 195 documentos en relación con el tipo de documento. El $45.1 \backslash \%$ de los documentos son artículos de revista, 40\\% son artículos de conferencia, un 7.2 $\ \%$ son revisiones de la literatura, mientras que un 5.1\\% forman capítulos de libros. El 2.1\\% son libros publicados y el $0.5 \backslash \%$ son revisiones de conferencias. Los resultados del análisis estadístico se exponen en la sección 3.1, como respuestas a las primeras 4 preguntas de investigación. A este conjunto se agregaron 3 artículos encontrados fuera de la consulta, considerados de interés para un análisis cualitativo por ser referentes de la situación en México, dando un total de 198 documentos.

Para el análisis cualitativo se realizó un filtrado de los documentos encontrados. Se eliminaron 37 documentos que no se refieren a tutores como herramientas tecnológicas, 54 documentos cuya contribución era en el área del cómputo afectivo y no en el área educativa, 26 documentos que no utilizan tutores afectivos, sino agentes afectivos para el aprendizaje mediado por computadora y 3 libros de fundamentos teóricos en relación a los sistemas tutores inteligentes. Finalmente, de los 75 documentos restantes, se hizo un análisis comparativo de 50 estudios, tomando en cuenta los indicadores de relevancia de la clasificación documental, el título y el resumen del artículo. En la Figura 3 se resume el proceso de exclusión de los registros encontrados.

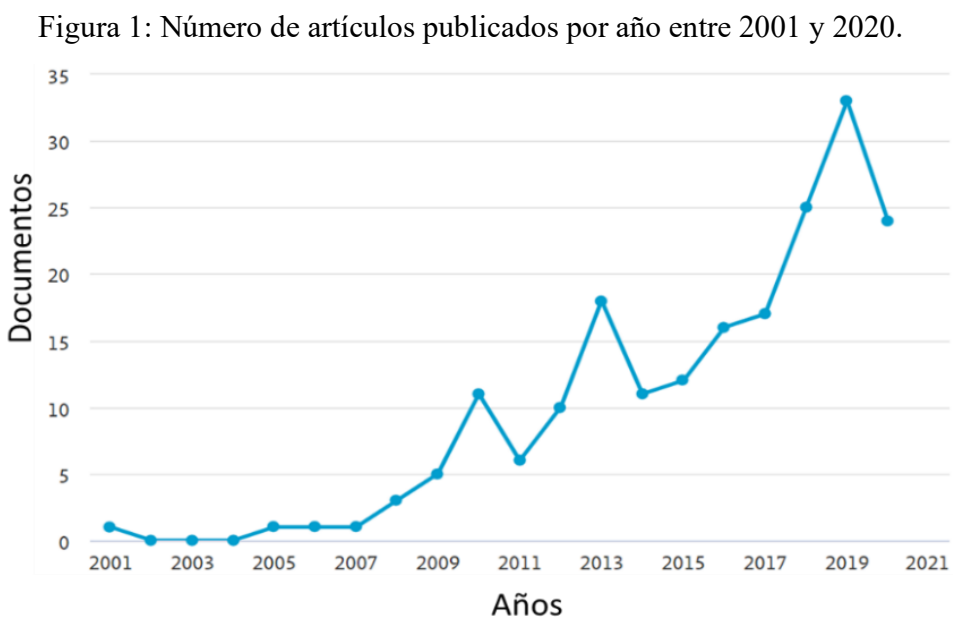

\subsection{Análisis bibliométrico}

Los resultados del análisis estadístico de la revisión sistemática sobre los sistemas tutores afectivos dan respuesta a las primeras 4 preguntas de investigación. A continuación, se describen las observaciones de dicho análisis.

¿Cuáles son las principales fuentes de publicación con relación a los ITS que integran las emociones en el proceso de aprendizaje?

Los 195 documentos provienen de diferentes revistas y editoriales, siendo las más importantes, con relación al número de aportaciones encontradas, (1) Lecture Notes In Computer Science Including Subseries Lecture Notes In Artificial Intelligence And Lecture Notes In Bioinformatics, (2) Computers And Education, (3) International Journal Of Artificial Intelligence In Education, (4) Computers In Human Behavior, (5) ACM International Conference Proceeding Series, (6) Ceur Workshop Proceedings, (7) Educational Psychology Review, (8) Frontiers In 
Psychology, (9) Journal Of Educational Psychology, (10) British Journal Of Educational Technology. La Figura 4 muestra la cantidad de documentos provenientes de las principales fuentes.

Figura 2: Comparativa de los tipos de publicación de los documentos analizados.

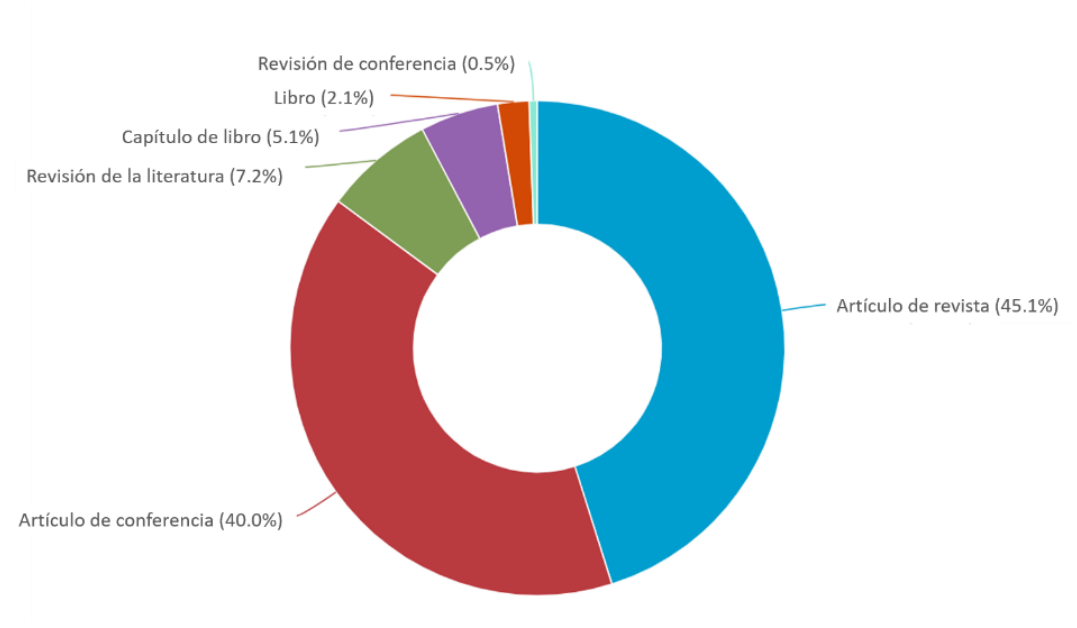

Figura 3: Diagrama de flujo del filtrado de documentos revisados.

\begin{tabular}{|c|c|}
\hline \multicolumn{2}{|c|}{ Documentos identificados en WoS, Scopus, ERIC y Dimensions ( $n=195)$} \\
\hline \multicolumn{2}{|c|}{$\begin{array}{l}\text { Inclusión de } 3 \text { artículos de investigación mexicana encontrados fuera } \\
\text { de la consulta original ( } n=198)\end{array}$} \\
\hline \multicolumn{2}{|c|}{$\begin{array}{l}\text { Exclusión de documentos que no hablan de sistemas inteligentes } \\
\qquad(\mathrm{n}=158)\end{array}$} \\
\hline \multicolumn{2}{|c|}{$\begin{array}{l}\text { Exclusión de documentos sin contribución a la investigación educativa } \\
\qquad(n=104)\end{array}$} \\
\hline Exclusión de documentos que se & efieren a agentes afectivos $(n=78)$ \\
\hline $\begin{array}{l}\text { Exclusión de libros sobre fundan } \\
\qquad(n=\end{array}$ & $\begin{array}{l}\text { entos de los tutores inteligentes } \\
75)\end{array}$ \\
\hline
\end{tabular}

Figura 4: Documentos por fuente de publicación.

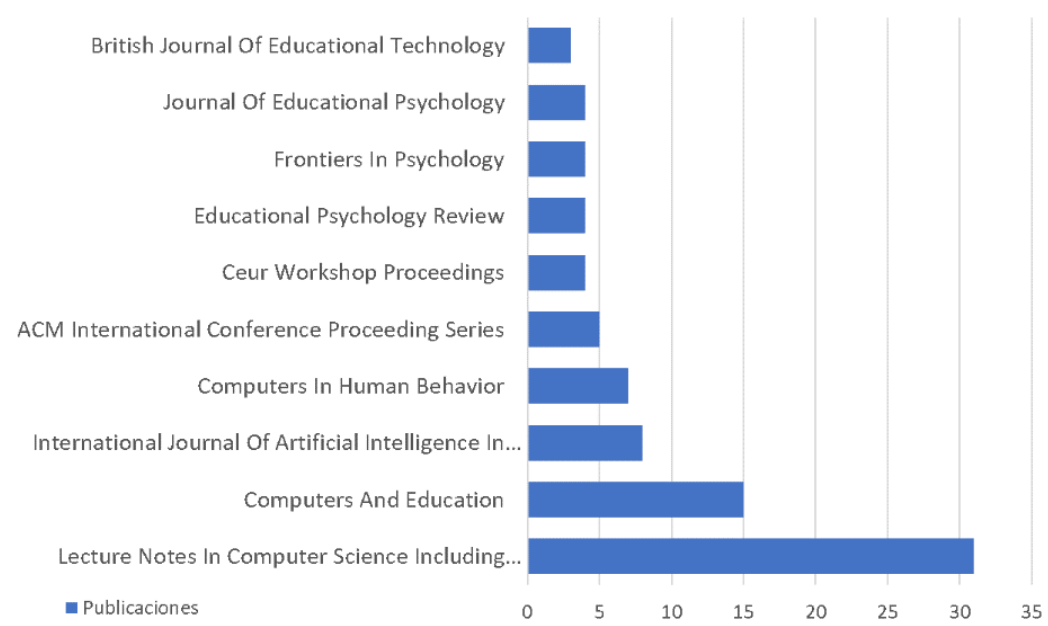


¿Quiénes son los principales autores en relación con el problema específico de los tutores inteligentes afectivos?

Uno de los objetivos de esta revisión de la literatura es identificar a los principales autores que abordan el problema de interés de la investigación. Después de hacer un análisis de los 195 documentos correspondientes al conjunto obtenido, se identificaron como autores más productivos aquellos mostrados en la Figura 5. Cabe destacar que en este análisis sólo se identifica la producción y no las citas de cada autor. Los 10 autores más productivos incluyen a (1) Arroyo, I., (2) Muldner, K., (3) Baker, R.S., (4) Azevedo, R., (5) Burleson, W., (6) Lester, J.C., (7) D’Mello, S., (8) Woolf, B.P, (9) Taub, M., y (10) Bosch, N.

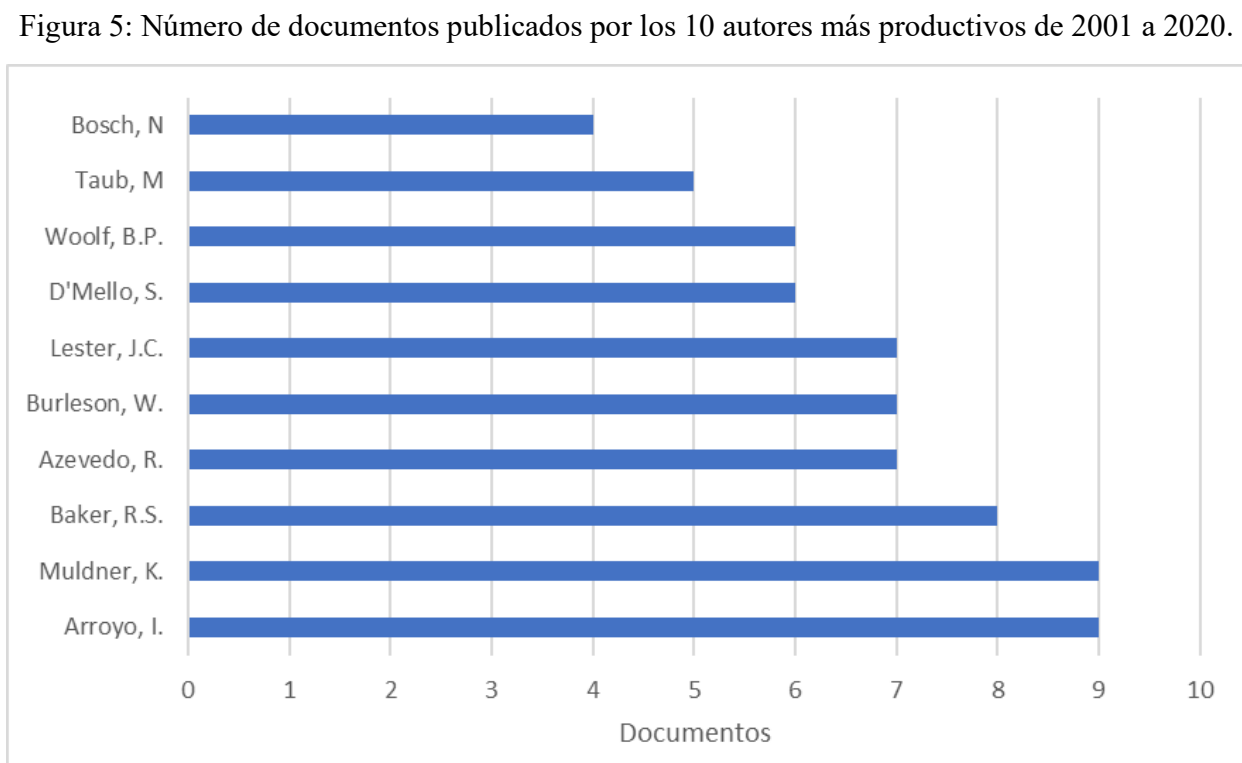

¿Cuáles son los conceptos más relevantes con relación a la evaluación del aprendizaje mediado por un ITS?

Se realizó un análisis de los resúmenes y palabras clave de los 195 documentos obtenidos para generar un mapa con los conceptos en común más relevantes y las relaciones entre ellos. La Figura 6 muestra un mapa de calor que señala la relevancia de cada concepto en relación al conjunto de documentos. Los conceptos de frustración y confusión, que de acuerdo con los métodos de identificación de emociones centradas en el aprendizaje son consideradas emociones negativas, así como expresión facial, que forma parte de los métodos de reconocimiento de emociones, aparecen entre los nodos más grandes. También se puede observar que las matemáticas son el único campo de aplicación que aparece de forma recurrente en los documentos.

¿Cuáles son las principales disciplinas en las que se ha hecho investigación sobre los ITS adaptativos a las emociones?

Los 195 documentos se clasificaron en relación al área de conocimiento del que proviene cada investigación. En la Figura 7 se muestra un comparativo entre las áreas y su producción científica alrededor de los ITS afectivos. El $41 \backslash \%$ de los documentos provienen del área de las ciencias computacionales, seguida por las ciencias sociales con un $27.2 \backslash \%$ y las matemáticas con un $10.11 \%$. En una cuarta instancia se encuentra la psicología con $9.21 \%$ y debajo las ingenierías con $5.21 \%$ y las artes y humanidades con $3.21 \%$. Una mínima parte de los documentos pertenece al área de negocios con $0.9 \backslash \%$, las ciencias de decisión, la economía y ciencias materiales, cada una con $0.6 \backslash \%$ y el $1.4 \backslash \%$ proveniente de otras áreas. 
Figura 6: Densidad de los principales conceptos obtenidos de los documentos analizados.

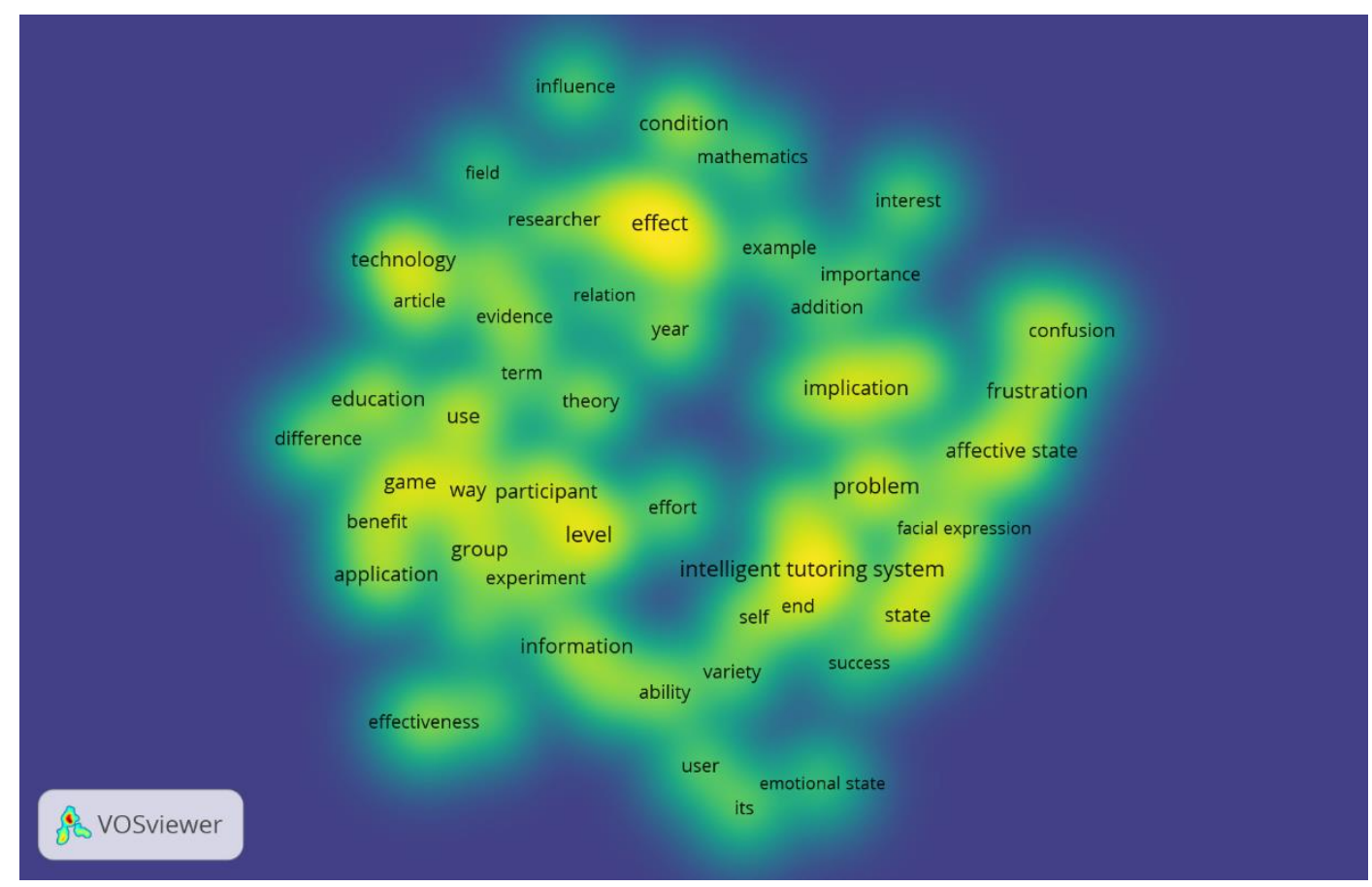

\subsection{Análisis cualitativo}

A continuación, se reportan los resultados del análisis cualitativo de los 75 documentos más relevantes en esta revisión de la literatura.

¿Cuáles son los hallazgos más relevantes en la integración de emociones dentro de los sistemas tutores inteligentes?

Con respecto a la inclusión del estado emocional en el aprendizaje mediado por tecnología, los estudios de investigación se han desarrollado dando soporte a dos grandes fases del proceso de integración.

\section{Identificación de las emociones en el aprendizaje}

La primera fase consiste en identificar las emociones persistentes y esporádicas que se presentan comúnmente durante la sesión de aprendizaje con un sistema tutor inteligente. Los fundamentos de la identificación de emociones en los tutores inteligentes son los de la teoría de las seis emociones básicas (ira, disgusto, miedo, alegría, tristeza y sorpresa) (Ekman y Keltner, 1997), y el modelo OCC (Ortony, Clore, y Collins, 1988) que contempla 14 emociones secundarias. En el contexto educativo se ha propuesto una clasificación de emociones académicas (Pekrun, 1992) que propone cuatro emociones positivas (alegría, esperanza, orgullo y alivio) y cinco negativas (aburrimiento, ansiedad, miedo y desesperanza). En 2010, D’Mello et al. identificaron 13 emociones que clasificaron en 3 grupos (D’Mello, Lehman, y Person, 2010). En la Tabla 1 se muestra la clasificación propuesta por D'Mello. 
Figura 7: Clasificación del conjunto de documentos en relación con el área de estudio.

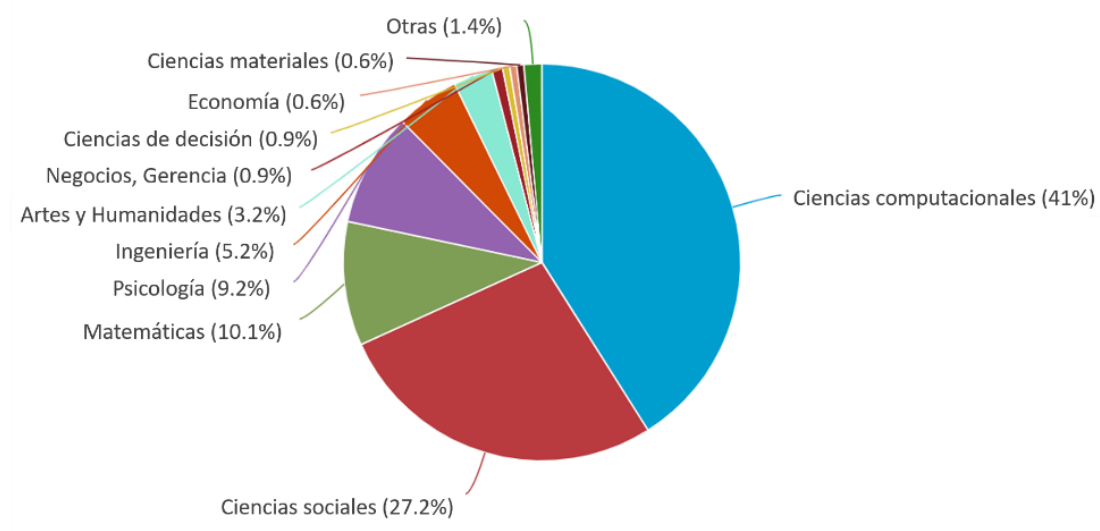

Tabla 1: Clasificación de emociones identificadas por D’Mello.

\begin{tabular}{|c|c|}
\hline Categoría & Emociones \\
\hline \multirow{3}{*}{ Rutinarias } & Aburrimiento \\
& Confusión \\
& Curiosidad \\
& Frustración \\
\hline \multirow{2}{*}{ Esporádicas } & Ansiedad \\
& Felicidad \\
\hline \multirow{2}{*}{ Excepcionales } & Rechazo \\
& Eureka \\
& Ira \\
& Disgusto \\
& Miedo \\
& Tristeza \\
& Sorpresa \\
\hline
\end{tabular}

No obstante, se suele referir al estado emocional en relación a su valor. Es decir, si la emoción se percibe como positiva o negativa, y a su vez si la emoción es activa o pasiva (Mayer, 2019; Russell, 1983). De manera similar, en (M. C. Duffy, Lajoie, Pekrun, y Lachapelle, 2020) se identificaron el placer, como la emoción positiva activa más recurrente, y la ansiedad, como la emoción negativa activa más recurrente, en un escenario de estudiantes de medicina. A su vez, en (Lehman, D’Mello, y Graesser, 2012) se trabaja la identificación de la confusión en periodos de interacción cortos con AutoTutor (A. C. Graesser y cols., 1999).

\section{Procesamiento de las emociones}

En cuanto al procesamiento automático de las emociones identificadas, la diversidad de métodos existentes en la literatura se divide en dos categorías. Aquellos que adquieren, utilizan y procesan el lenguaje natural del estudiante durante la sesión; y aquellos que toman otros indicadores presentes en el proceso interactivo a corto plazo.

Desde el punto de vista computacional existen muchas técnicas y herramientas para adquirir información sobre el estado emocional de una persona. El cómputo afectivo es una línea de investigación dentro de las ciencias computacionales en la que se analiza y se desarrolla tecnología cuyo comportamiento es influenciado por las emociones de los usuarios. Particularmente, en el desarrollo de ITS afectivos se ha hecho uso de indicadores directos, como cuestionarios, e indirectos como seguimiento de gestos y movimientos, para adquirir información relevante, tanto antes, como durante y después de las sesiones interactivas que un estudiante tiene con un sistema tutor. Más allá de las diferentes categorías en cuanto a los datos adquiridos, Mahmoud hace una clasificación de las herramientas de adquisición utilizadas en el contexto de 
los ITS que distingue aquellas que adquieren datos de comportamiento de las que adquieren datos psicológicos, ya sea utilizando sensores o métodos de auto reporte de datos (Mahmoud, 2019). Sobresalen los métodos de auto reporte por su bajo costo de implementación. Se ha hecho uso de herramientas verbales como cuestionarios y entrevistas o indicadores independientes del lenguaje, como escalas Likert. Java sensei (Cabada, Estrada, Hernández, y Bustillos, 2015) y SentBuk (Martin, Ortigosa, y Carro, 2012) hacen uso del análisis de comentarios de retroalimentación para detectar las emociones del usuario después de las sesiones interactivas.

Por otro lado, el uso de sensores como los electroencefalogramas, electrocardiogramas, seguidores del ojo e incluso cámaras de video, han permitido innovar en el análisis de la información emocional de los estudiantes. El reconocimiento de emociones a través del análisis facial se ha utilizado recientemente con la ventaja de la facilidad de implementación con teléfonos inteligentes o computadoras portátiles. El auge de la inteligencia artificial ha permitido el uso de modelos sofisticados en el análisis de emociones. El análisis del discurso, ya sea textual o verbal, utilizando sistemas de aprendizaje de máquina ha sido aplicado dentro de muchos dominios, abriendo una oportunidad al campo de los ITS.

Se revisaron 50 documentos para un análisis en relación con la integración de las emociones al escenario de aprendizaje. En la Tabla 2 se muestra un resumen de los resultados del análisis. 


\begin{tabular}{|c|c|c|c|c|c|c|}
\hline Referencia & Modelo de emociones & Emociones relevantes en el estudio & $\begin{array}{c}\text { Etapas de } \\
\text { procesamiento de } \\
\text { emociones } \\
\end{array}$ & Técnicas de procesamiento & Evaluación & $\begin{array}{r}\text { Intervención } \\
\text { del docente }\end{array}$ \\
\hline (Alyuz, 2016) & Modelo OCC & $\begin{array}{l}\text { euforia, calma, aburrimiento y } \\
\text { confusión }\end{array}$ & Identificación & $\begin{array}{l}\text { Reconocimiento facial de } \\
\text { emociones y seguimiento de } \\
\text { interacción }\end{array}$ & Automático & No \\
\hline (Andres y cols., 2019) & $\begin{array}{l}\text { Modelo de D'Mello y } \\
\text { Graesser (2012) }\end{array}$ & aburrimiento y gozo & Análisis & Libre de sensores & Cuestionarios & No \\
\hline $\begin{array}{c}\text { (Arguedas y cols., } \\
\text { 2016) } \\
\end{array}$ & Plutchick & $\begin{array}{l}\text { Ira, disgusto, miedo, alegría, tristeza, } \\
\text { sorpresa y confianza }\end{array}$ & Identificación & Análisis semántico de textos & Cuestionarios & No \\
\hline (Arguel y cols., 2019) & $\begin{array}{c}\text { Pekrun, emociones } \\
\text { epistémicas (D'Mello et al. } \\
2010) \\
\end{array}$ & confusión & Identificación & $\begin{array}{l}\text { Reconocimiento facial de } \\
\text { emociones y seguimiento de } \\
\text { interacción }\end{array}$ & Auto reporte & No \\
\hline (Arroyo y cols., 2014) & Ekman & confianza, interés, frustración & Identificación & Libre de sensores & Auto reporte & No \\
\hline (Arroyo y cols., 2016) & No se especifica & $\begin{array}{l}\text { frustración, confianza, ansiedad, } \\
\text { interés, aburrimiento y euforia }\end{array}$ & Análisis y Adaptación & Libre de sensores & Auto reporte & No \\
\hline $\begin{array}{c}\text { (Ashwin y Guddeti, } \\
\text { 2020) }\end{array}$ & $\begin{array}{c}\text { Ekman y emociones } \\
\text { epistémicas }\end{array}$ & $\begin{array}{l}\text { compromiso, felicidad, confusión, } \\
\text { aburrimiento }\end{array}$ & $\begin{array}{c}\text { Identificación, Análisis } \\
\text { y Adaptación }\end{array}$ & $\begin{array}{c}\text { Reconocimiento facial, postura } \\
\text { corporal y gestos de la mano }\end{array}$ & Cuestionarios & No \\
\hline (Aslan y cols., 2019) & $\begin{array}{c}\text { Modelo circunflejo de } \\
\text { emociones (Russel, 1980) }\end{array}$ & $\begin{array}{c}\text { emociones positivas, negativas y } \\
\text { neutrales (en general) }\end{array}$ & Identificación & Reconocimiento facial & $\begin{array}{l}\text { Etiquetado por } \\
\text { expertos }\end{array}$ & No \\
\hline $\begin{array}{c}\text { (Baldassarri y cols., } \\
\text { 2015) }\end{array}$ & Ekman & $\begin{array}{c}\text { sorpresa, alegría, ira, disgusto, temor, } \\
\text { tristeza }\end{array}$ & Identificación & $\begin{array}{c}\text { Reconocimiento facial de } \\
\text { emociones }\end{array}$ & Cuestionarios & Sí \\
\hline $\begin{array}{c}\text { (Bosch y D'Mello, } \\
\text { 2013) }\end{array}$ & No se especifica & $\begin{array}{l}\text { emociones que propicien el } \\
\text { aprendizaje (no se especifica) }\end{array}$ & $\begin{array}{c}\text { Identificación, Análisis } \\
\text { y Adaptación }\end{array}$ & Reconocimiento facial & Cuestionarios & No \\
\hline (Cabada y cols., 2015) & Ekman & alegría, sorpresa y escepticismo & Adaptación & Reconocimiento facial & Automático & No \\
\hline (Chao y cols., 2014) & Ekman y Pekrun & $\begin{array}{c}\text { alegría, tristeza, ira, sorpresa, } \\
\text { frustración, incertidumbre, disgusto, } \\
\text { temor }\end{array}$ & ıdentincacion & Análisis semántico de textos & $\begin{aligned} \text { RBIE v.2 } & \text { Cuesuvinuvo }\end{aligned}$ & $29-\underset{1}{2021}$ \\
\hline $\begin{array}{c}\text { (Chaouachi y Frasson, } \\
\text { 2012) }\end{array}$ & No se especifica & $\begin{array}{l}\text { interés, alegría, confianza, relajación, } \\
\text { confusión, frustración, aburrimiento, } \\
\text { desinterés }\end{array}$ & Identificación y Análisis & Electroencefalograma & Auto reporte & No \\
\hline (D'Mello y cols., 2010) & $\begin{array}{l}\text { Ekman y emociones } \\
\text { epistémicas }\end{array}$ & $\begin{array}{l}\text { ira, disgusto, miedo, alegría, tristeza, } \\
\text { sorpresa, ansiedad, aburrimiento, } \\
\text { confusión, curiosidad, frustración }\end{array}$ & Identificación & Reconocimiento facial & Auto reporte & No \\
\hline $\begin{array}{l}\text { (Duffy y Azevedo, } \\
2015)\end{array}$ & No se especifica & motivación & Análisis & Libre de sensores & Cuestionarios & No \\
\hline
\end{tabular}




\begin{tabular}{|c|c|c|c|c|c|c|}
\hline $\begin{array}{l}\text { (Fotopolou y cols., } \\
\text { 2020) }\end{array}$ & $\begin{array}{c}\text { Competencias sociales y } \\
\text { emocionales }\end{array}$ & automotivación, autoestima & Análisis y Adaptación & Seguimiento de interacción & Automático & No \\
\hline (Ghaleb y cols., 2019) & Teoría de Flujo & aburrimiento, compromiso, frustración & Identificación & Seguimiento de interacción & Auto reporte & No \\
\hline (Graesser y cols., 2017) & $\begin{array}{l}\text { Modelo de D'Mello y } \\
\text { Graesser (2012) }\end{array}$ & confusión & Análisis y Adaptación & Diálogo afectivo (triálogo) & Automático & No \\
\hline $\begin{array}{l}\text { (Grafsgaard y cols., } \\
\text { 2013) }\end{array}$ & No se especifica & compromiso y frustración & Identificación & Reconocimiento facial & $\begin{array}{l}\text { Cuestionarios y } \\
\text { automático }\end{array}$ & No \\
\hline $\begin{array}{l}\text { (Gudino-Penaloza y } \\
\text { cols., 2009) }\end{array}$ & No se especifica & gozo, compromiso, interés & Adaptación & Libre de sensores & Cuestionarios & No \\
\hline (Harley y cols., 2016) & Pekrun & Gozo y aburrimiento & Identificación & $\begin{array}{c}\text { Seguimiento ocular, análisis de } \\
\text { audio e interacción verbal }\end{array}$ & Auto reporte & No \\
\hline $\begin{array}{l}\text { (Hull y du Boulay, } \\
\text { 2015) }\end{array}$ & $\begin{array}{l}\text { Teoría de la motivación de } \\
\text { Schunk (2007) }\end{array}$ & motivación & Análisis & Seguimiento de interacción & Cuestionarios & No \\
\hline (Hussain y cols., 2011) & No se especifica & $\begin{array}{l}\text { frustración, confusión, compromiso, } \\
\text { fusto, sorpresa, aburrimiento, } \\
\text { curiosidad }\end{array}$ & Identificación & \begin{tabular}{|} 
Electroencefalograma, \\
electrocardiograma, \\
electromiograma, respiración y \\
respuesta galvánica de la piel
\end{tabular} & Auto reporte & No \\
\hline (Jimenez y cols., 2018) & No se especifica & motivación & Adaptación & Diálogo afectivo & Escala Likert & No \\
\hline (Joshi y cols., 2019) & No se especifica & confusión, interés, confianza & Identificación y Análisis & $\begin{array}{l}\text { Reconocimiento facial de } \\
\text { emociones }\end{array}$ & Automático & No \\
\hline (Lajoie y cols., 2019) & $\begin{array}{l}\text { Ekman, Pekrun y teoría de la } \\
\text { motivación de Schunk (2007) }\end{array}$ & $\begin{array}{c}\text { Ira, disgusto, felicidad, tristeza, miedo } \\
\text { y sorpresa }\end{array}$ & Análisis & Reconocimiento facial & Automático & No \\
\hline (Lehman y cols., 2012) & Emociones epistémicas & confusión & & Libre de sensores & \multicolumn{2}{|c|}{ Auto RBIE v.29-2021 } \\
\hline (Leyzberg y cols., 2014) & No se especifica & compromiso & Adaptación & Diálogo afectivo & Escala Likert & No \\
\hline (Liu y cols., 2013) & No se especifica & confusión y frustración & Identificación y Análisis & $\begin{array}{c}\text { Reconocimiento facial, postura } \\
\text { corporal y seguimiento de } \\
\text { interacción }\end{array}$ & Automático & No \\
\hline (Long y cols., 2019) & Emociones epistémicas & confusión & Adaptación & Libre de sensores & Auto reporte & No \\
\hline (Long y Aleven, 2017) & No se especifica & gozo & Identificación & Libre de sensores & Cuestionarios & No \\
\hline (Magner y cols., 2010) & No se especifica & euforia, aburrimiento, entretenimiento & Análisis & Libre de sensores & Escala Likert & No \\
\hline $\begin{array}{l}\text { (Marco-Gimenez y } \\
\text { cols., 2016) }\end{array}$ & No se especifica & satisfacción y motivación & $\begin{array}{c}\text { Identificación, Análisis } \\
\text { y Adaptación }\end{array}$ & $\begin{array}{l}\text { Electroencefalograma y } \\
\text { reconocimiento facial }\end{array}$ & Automático & No \\
\hline
\end{tabular}




\begin{tabular}{|c|c|c|c|c|c|c|}
\hline $\begin{array}{l}\text { (McQuiggan y cols., } \\
\text { 2008) }\end{array}$ & $\begin{array}{l}\text { Ekman y emociones } \\
\text { epistémicas }\end{array}$ & \begin{tabular}{|c|}
$\begin{array}{c}\text { ira, ansiedad, aburrimiento, gusto, } \\
\text { confusión, entusiasmo, miedo, flujo, } \\
\text { frustración y tristeza }\end{array}$ \\
\end{tabular} & $\begin{array}{l}\text { Identificación y } \\
\text { Adaptación }\end{array}$ & Libre de sensores & Auto reporte & No \\
\hline (Muldner y cols., 2015) & Pekrun & aburrimiento y falta de entusiasmo & Análisis y adaptación & Libre de sensores & $\begin{array}{l}\text { Cuestionarios, } \\
\text { auto reporte y } \\
\text { escala Likert }\end{array}$ & No \\
\hline (Muñoz y cols., 2010) & Pekrun & emociones de logro (motivación) & Identificación & Libre de sensores & Cuestionarios & No \\
\hline $\begin{array}{c}\text { (Padron-Rivera y cols., } \\
\text { 2018) }\end{array}$ & $\begin{array}{l}\text { Modelo de D'Mello y } \\
\text { Graesser (2012) }\end{array}$ & frustración y confusión & Identificación & Reconocimiento facial & Automático & No \\
\hline (Pardos y cols., 2013) & $\begin{array}{c}\text { Diversas teorías y } \\
\text { observaciones empíricas }\end{array}$ & $\begin{array}{l}\text { aburrimiento, frustración, } \\
\text { concentración, confusión }\end{array}$ & Análisis & $\begin{array}{l}\text { Seguimiento de interacción, } \\
\text { reconocimiento facial y } \\
\text { corporal }\end{array}$ & Automático & Sí \\
\hline $\begin{array}{l}\text { (Pedroza-Méndez y } \\
\text { cols., 2019) }\end{array}$ & No se especifica & motivación & Adaptación & Libre de sensores & Cuestionarios & Sí \\
\hline (Pham y Wang, 2018) & No se especifica & $\begin{array}{l}\text { aburrimiento, confusión, curiosidad, } \\
\text { frustración, felicidad }\end{array}$ & Identificación & $\begin{array}{l}\text { Reconocimiento facial y } \\
\text { fotopletismografía }\end{array}$ & Escala Likert & No \\
\hline (Price y cols., 2018) & Pekrun & confusión, frustración, ira, ansiedad & Análisis y Adaptación & Libre de sensores & Auto reporte & No \\
\hline (Richey y cols., 2019) & $\begin{array}{l}\text { Modelo de D'Mello y } \\
\text { Graesser (2012), }\end{array}$ & confusión y frustración & Análisis & Seguimiento de interacción & $\begin{array}{l}\text { Etiquetado por } \\
\text { expertos }\end{array}$ & Sí \\
\hline (Rodrigo y cols., 2012) & No se especifica & aburrimiento, confusión, compromiso & Adaptación & Diálogo afectivo & $\begin{array}{c}\text { Cuestionarios y } \\
\text { escala Likert }\end{array}$ & No \\
\hline (Ruiz y cols., 2020) & $\begin{array}{l}\text { Pekrun, modelo de Arroyo } \\
(2009)\end{array}$ & interés y frustración & Identificación & Seguimiento de interacción & Escala Likert & No \\
\hline (Strain y cols., 2011) & $\begin{array}{c}\text { Emociones centradas en el } \\
\text { aprendizaje }\end{array}$ & frustración, ansiedad y aburrimiento & Análisis y adaptación & Reconocimiento facial & Auto reporte & No \\
\hline (Taub y cols., 2020) & $\begin{array}{c}\text { Pekrun, modelo de D'Mello y } \\
\text { Graesser (2012) }\end{array}$ & alegría, confusión y frustración & Identificación y análisis & $\begin{array}{l}\text { Reconocimiento facial y } \\
\text { actividad electro dérmica }\end{array}$ & Cuestionarios & No \\
\hline (Vail y cols., 2016) & No se especifica & frustración y compromiso & Identificación y Análisis & $\begin{array}{l}\text { Reconocimiento facial y } \\
\text { corporal }\end{array}$ & $\begin{array}{l}\text { Cuestionarios, } \\
\text { auto reporte }\end{array}$ & No \\
\hline (Wang y Ling, 2018) & Ekman & emociones negativas (no especifica) & $\begin{array}{c}\text { Identificación, Análisis } \\
\text { y Adaptación }\end{array}$ & $\begin{array}{c}\text { Análisis semántico de textos y } \\
\text { Reconocimiento facial de } \\
\text { emociones }\end{array}$ & Cuestionarios & No \\
\hline (Woolf y cols., 2010) & Ekman & $\begin{array}{c}\text { motivación, confianza, frustración, } \\
\text { euforia }\end{array}$ & Adaptación & Libre de sensores & Cuestionarios & No \\
\hline
\end{tabular}


(Zakharov y cols., $\quad$ No se especifica 2007) estrés, ansiedad y frustración
Identificación
Reconocimiento facial, señales fisiológicas

Automático 
Como resultado de la comparación entre estos 50 trabajos se puede observar que la investigación tiene una tendencia hacia el proceso de identificación de las emociones que se presentan durante el uso de recursos como los ITS. Un total de 28 trabajos abordan el problema de identificación de las emociones que se presentan cuando un estudiante hace uso de la herramienta, mientras que el proceso de adaptación de la interacción, en cuanto al diseño instruccional, los recursos educativos y los niveles de dificultad de tareas, ha sido parte de 19 trabajos. De igual forma, 23 trabajos abordan el análisis del significado e impacto de estas emociones en el proceso de aprendizaje.

Por otro lado, la evaluación de la efectividad de la herramienta, con relación a la integración de emociones, sigue siendo a través del uso de cuestionarios y auto reportes, formando parte de 32 trabajos. Un total de 11 estudios utilizan una evaluación automática a partir de herramientas de software, 7 trabajos utilizan la escala Likert y sólo 2 estudios hacen uso de la evaluación por expertos.

A pesar de que en algunos trabajos no se reporta la teoría de emociones considerada como fundamento de la integración del estado afectivo, los modelos de Ekman y Pekrun siguen siendo referentes. En (Ghaleb y cols., 2019) se introduce la Teoría del Flujo (Nakamura y Csikszentmihalyi, 2014) en la que se modelan las emociones a partir de 3 estados principales (frustración, interés y aburrimiento), entre los cuales el estudiante transita a medida que realiza una tarea cognitiva.

Sólo 4 de los trabajos del conjunto analizado tiene una intervención directa del docente.

\section{¿Cuál es el efecto en el proceso de aprendizaje del uso de los sistemas tutores afectivos?}

Con base en una lectura profunda de los documentos seleccionados para un análisis cualitativo, aunada a los resultados estadísticos, especialmente al análisis de conceptos relevantes y resúmenes, se puede observar una carencia de reporte de la efectividad con respecto al área educativa con relación al uso de ITS afectivos. Aún en los últimos años, los estudios destacan la eficacia de los métodos computacionales por sobre el efecto en los objetivos de aprendizaje. Algunos trabajos como el de Price en (Price y cols., 2018), describen el papel que juegan las emociones negativas durante el uso de un tutor inteligente, pero no lo relacionan con el desempeño educativo. De igual forma, la evaluación de la efectividad de los ITS afectivos, más allá del aspecto tecnológico, se ha dado en relación con la percepción de los usuarios sobre los mismos. Algunos autores han reportado la satisfacción de los estudiantes y otros resultados de evaluación de usabilidad de software como evidencia del efecto positivo para los usuarios. Mayer (Mayer, 2019) declara que no hay suficiente evidencia para asegurar la efectividad de la adaptación de las emociones en los recursos e-learning en cuanto al objetivo de aprendizaje.

\section{¿Cuál es el estado de la investigación de los sistemas tutores afectivos en México?}

Dentro de las bases de datos consultadas, el porcentaje de registros que provienen de países latinoamericanos es significativamente bajo. No obstante, en la revisión sobre las tendencias de los sistemas tutores inteligentes de Han (Han, Zhao, Jiang, Oubibi, y Hu, 2019), Chile es el país latinoamericano con mayor producción en la investigación de los ITS ubicado en el lugar 12 de 50 países en cuanto al desarrollo de la investigación en este ámbito. Argentina ocupa la posición 15, Brasil en el lugar 19, Costa Rica en el lugar 22, y México está en el lugar 34, siendo el último país latinoamericano que aparece.

Entre los trabajos de investigación en México que destacan en las bases de datos, específicamente en el área de los sistemas tutores afectivos, el más reconocido es Fermat (Cabada y cols., 2012), un tutor de matemáticas que se encuentra dentro de una red social que utiliza datos verbales y no verbales para identificar las emociones presentes en la sesión interactiva y a su vez da respuesta a través de un agente afectivo. En la Figura 8 se muestra la interfaz de Fermat cuando 
un agente afectivo está dando una instrucción guiada al estudiante. En (Jimenez y cols., 2018), los autores desarrollan un sistema de retroalimentación con características afectivas que se integra a un tutor inteligente para la enseñanza de programación orientada a objetos, con la intención de analizar el efecto en la confianza que tienen los estudiantes cuando reciben mensajes positivos durante el proceso interactivo. Gudino-Penaloza (Gudino-Penaloza y cols., 2009), implementa la adaptación de un tutor inteligentes a las necesidades de los estudiantes de preparatoria en un curso de probabilidad y estadística. Padrón-Rivera (Padrón-Rivera, Rebolledo-Mendez, Parra, y HuertaPacheco, 2016), realiza un análisis de la relación entre las unidades de acción y los estados afectivos que presentan los estudiantes de un curso de matemáticas guiado por un tutor inteligente. En (Pedroza-Méndez y cols., 2019), los autores hacen uso de técnicas de gamificación en el diseño de un tutor inteligente de apoyo en la resolución de problemas de álgebra, con el objetivo de hacer más placentera la sesión interactiva y al mismo tiempo reducir la carga cognitiva del estudiante.

Figura 8. Interfaz de Fermat (Cabada y cols., 2012)

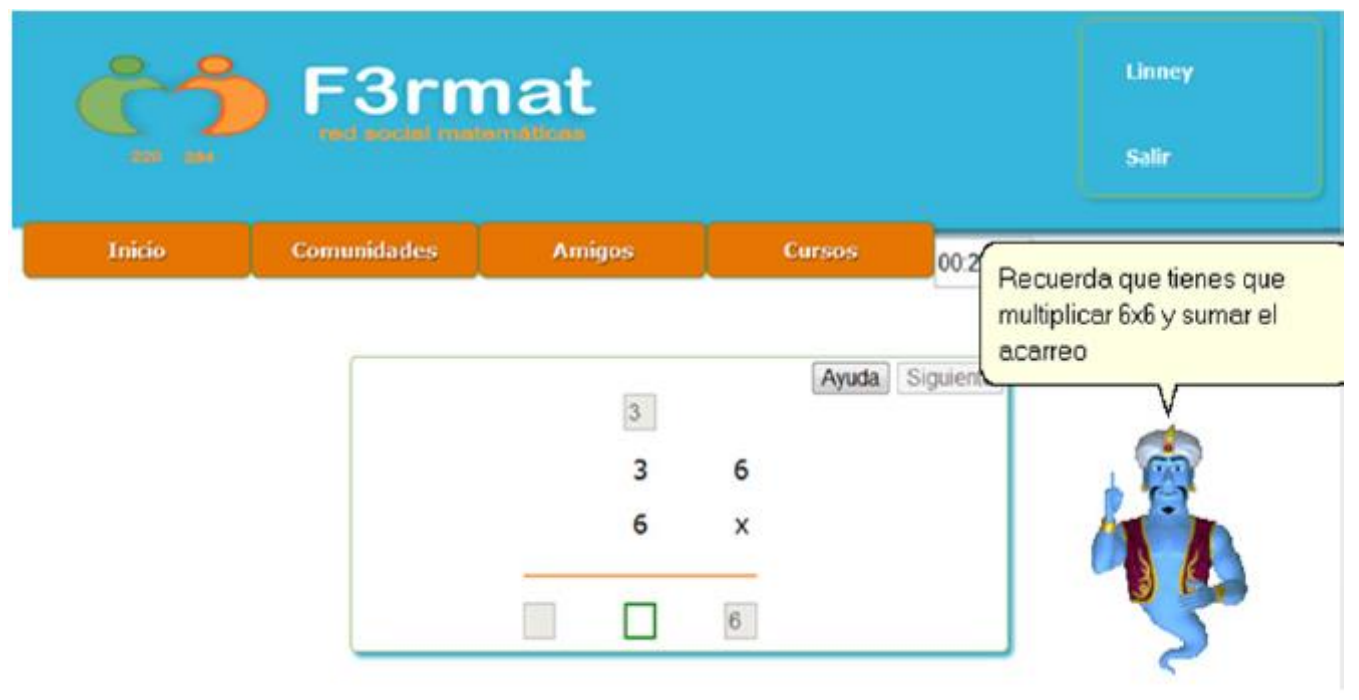

\section{Conclusiones}

De la primera etapa de esta revisión de la literatura sobre los ITS afectivos se obtuvieron 195 documentos utilizando como criterios de búsqueda los conceptos learning outcomes, intelligent tutoring systems, y emotions. En los resultados del análisis bibliométrico de este conjunto de documentos se puede observar que el estudio e interés de la comunidad científica sobre los ITS afectivos es creciente, aunque tuvo bajas en 2011 y 2014, pero que en 2019 tiene un alto porcentaje de producción. Tomando en cuenta la situación mundial actual en relación con el COVID-19 y los cambios en las prácticas educativas de la modalidad presencial a la modalidad a distancia, se espera que la investigación relacionada a la mediación tecnológica del proceso de enseñanza aumente, incluyendo el desarrollo de ITS y de aquellos que integran el estado afectivo del estudiante.

Las fuentes de información de los documentos indexados en las bases de datos utilizadas son principalmente revistas relacionadas al desarrollo computacional, lo que tiene sentido ya que el análisis estadístico muestra que cerca de un $50 \backslash \%$ de los documentos provienen de esta disciplina. Sin embargo, también hay relevancia en las revistas con enfoque pedagógico, por lo que existe investigación importante no sólo en el desarrollo de las herramientas sino en la fundamentación del dominio de aplicación.

En el análisis de conceptos relevantes aparecen las emociones negativas de frustración y confusión. Como única emoción positiva aparece la palabra interés en un nodo relativamente 
pequeño. Así mismo, en el análisis cualitativo se identificó que 16 trabajos hacen referencia al aburrimiento como emoción relevante. Esto hace notar la existencia de un área de oportunidad en relación al análisis de las emociones positivas orientadas al aprendizaje. En menor cantidad, emociones positivas como la alegría, motivación, sorpresa e interés han sido de interés para su identificación y procesamiento dentro de los ITS. Esto refleja lo dicho por Mayer (Mayer, 2019), en referencia a que existe una necesidad de interpretación de la relación del estado emocional con respecto al aprendizaje, dado que aún las investigaciones más recientes se han enfocado en la mejora de los métodos computacionales de adquisición y análisis de emociones dentro de un contexto educativo y no llegan a una interpretación pedagógica. No obstante, se encontró que la comunidad científica tiene un interés en disminuir las emociones negativas en el aprendizaje a través de estrategias mediadas por un ITS, como la investigación de Jimenez y colegas en (Jimenez y cols., 2018), quienes fomentan la motivación a partir de la retroalimentación positiva de forma directa durante la interacción con el estudiante, denominando a esta estrategia como diálogo afectivo. En este mismo sentido, la teoría del flujo de emociones relaciona el desempeño de un individuo con el nivel de compromiso que presenta al ejecutar una tarea (Nakamura y Csikszentmihalyi, 2014). Esta teoría se ha aplicado dentro del desarrollo de entornos virtuales, y señala que hay una zona de flujo en la que una persona alcanza un nivel óptimo de productividad en la ejecución de una tarea, encontrando un equilibrio en el nivel de ansiedad, de forma que el individuo no presenta frustración, pero no llega a caer en aburrimiento. A pesar de que se ha determinado que en el proceso de aprendizaje intervienen un factor cognitivo, afectivo y metacognitivo (Taub y cols., 2020), no hay una gran cantidad de estudios que reporten la utilidad de la integración de las emociones con respecto a objetivos de aprendizaje específicos.

Finalmente, los resultados de la revisión sistemática hacen evidente un área de oportunidad dentro de los países latinoamericanos para aplicar y reportar la efectividad del uso de los ITS en general. Tomando en cuenta el vacío que existe con relación a la utilidad de estas herramientas para el aprendizaje, se requiere evidencia contextual que tome en cuenta las limitaciones y retos de la educación mediada por tecnologías en Latinoamérica, como son la inequidad de acceso a la tecnología y la falta de alfabetización tecnológica de la población.

\section{Referencias}

Alyuz, N. (2016). Shaping the future of education with empathic companions. En ERM4CT 2016 - Proceedings of the 2nd Workshop on Emotion Representations and Modelling for Companion Systems (pp. 1-2). doi: 10.1145/3009960.3009964. Descargado de https://www.scopus.com/inward/record.uri?eid=2-s2.0-

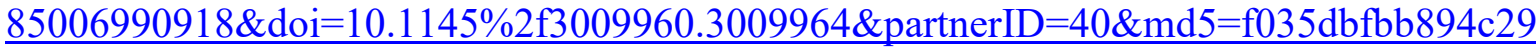
$\underline{3 \mathrm{c} 531607 \mathrm{e} 18 \mathrm{daae} 63}$

Andres, J., Paquette, L., Ocumpaugh, J., Jiang, Y., Baker, R., Karumbaiah, S., ..., \& Biswas, G. (2019). Affect sequences and learning in Betty's brain. En ACM International Conference Proceeding Series (pp. 383-390). doi: 10.1145/3303772.3303807. Descargado de https://www.scopus.com/inward/record.uri?eid=2-s2.0$\underline{85062792861 \& \text { doi }=10.1145 \% 2 \mathrm{f} 3303772.3303807 \& \text { partnerID }=40 \& \mathrm{md} 5=\mathrm{a} 0 \mathrm{c} 7 \mathrm{ad} 0 \mathrm{f} 37 \mathrm{adba} 9}$ $\underline{3 \mathrm{ccef} 35385237 \mathrm{c} 04 \mathrm{f}}$

Arguedas, M., Casillas, L., Xhafa, F., Daradoumis, T., Pena, A., \& Caballe, S. (2016). A FuzzyBased Approach for Classifying Students' Emotional States in Online Collaborative Work. En Proceedings - 2016 10th International Conference on Complex, Intelligent, and Software Intensive Systems, CISIS 2016 (pp. 61-68). doi: 10.1109/CISIS.2016.141. Descargado de https://www.scopus.com/record/display.uri?eid=2-s2.0- 
$\underline{85011028481 \& \text { doi }=10.1109 \% 2 \mathrm{fCISIS} .2016 .141 \& \text { origin=inward\&txGid=e6f143afd097ce7 }}$ c8c2a2f221edc8bba

Arguel, A., Lockyer, L., Chai, K., Pachman, M., \& Lipp, O. (2019). Puzzle-solving activity as an indicator of epistemic confusion. Frontiers in Psychology, 10(JAN). doi: 10.3389/fpsyg.2019.00163. Descargado

https://www.scopus.com/record/display.uri?eid=2-s2.0-

$\underline{85061061370 \& \text { doi }=10.3389 \% 2 \mathrm{ffpsyg} .2019 .00163 \& \text { origin }=\text { inward\&txGid=b7d1256f2e } 7 \mathrm{ba}}$ 2ffce8f9a6ecaf3704d

Arroyo, I., Schultz, S., Wixon, N., Muldner, K., Burleson, W., \& Woolf, B. (2016). Addressing affective states with empathy and growth mindset. En CEUR Workshop Proceedings (Vol. 1618). [GS Search]

Arroyo, I., Woolf, B. P., Burelson, W., Muldner, K., Rai, D., Tai, M. (2014, diciembre). A Multimedia Adaptive Tutoring System for Mathematics that Addresses Cognition, Metacognition and Affect. International Journal of Artificial Intelligence in Education, 24(4), 387-426. doi: 10.1007/s40593-014-0023-y. Descargado 2020-08-14, de http://link.springer.com/10.1007/s40593-014-0023-y

Ashwin, T., \& Guddeti, R. (2020). Impact of inquiry interventions on students in e-learning and classroom environments using affective computing framework. User Modeling and UserAdapted Interaction. doi: 10.1007/s11257-019-09254-3. Descargado de https://www.scopus.com/inward/record.uri?eid=2-s2.0$85077538149 \&$ doi $=10.1007 \% 2$ fs $11257-019-09254-$ $3 \&$ partnerID $=40 \& \mathrm{md} 5=5 \mathrm{e} 581 \mathrm{dd} 6 \mathrm{~d} 58 \mathrm{~d} 2 \mathrm{aed} 5 \mathrm{a} 84 \mathrm{e} 38 \mathrm{bfe} 1 \mathrm{bf} 120$

Aslan, S., Okur, E., Alyuz, N., Esme, A., \& Baker, R. (2019). Human expert labeling process: Valence-arousal labeling for students' affective states. Advances in Intelligent Systems and Computing, 804, 53-61. doi: 10.1007/978-3-319-98872-6 7. Descargado de https://www.scopus.com/inward/record.uri?eid=2-s2.0$85057307096 \&$ doi $=10.1007 \% 2 \mathrm{f} 978-3-319-98872-$ 6 7\&partnerID $=40 \& \mathrm{md} 5=56 \mathrm{f} 53 \mathrm{a} 28 \mathrm{bfde} 112946 \mathrm{f} 2 \mathrm{fd} 47 \mathrm{dd} 0 \mathrm{~d} 64 \mathrm{f} 7$

Baldassarri, S., Hupont, I., Abadía, D., \& Cerezo, E. (2015). Affective-aware tutoring platform for interactive digital television. Multimedia Tools and Applications, 74(9), 3183-3206. doi: 10.1007/s11042-013-1779-z. Descargado de https://www.scopus.com/record/display.uri?eid=2-s2.0$84928376617 \&$ doi $=10.1007 \% 2 \mathrm{fs} 11042-013-1779-$ $\underline{\text { z\&origin }}=$ inward\&txGid $=8593 \mathrm{a} 75198 \mathrm{aa} 04 \mathrm{~d} 87 \mathrm{~d} 31318 \mathrm{aaa} 257 \mathrm{~b} 70$

Bosch, N., \& D’Mello, S. (2013). Programming with your heart on your sleeve: Analyzing the affective states of computer programming students. Lecture Notes in Computer Science (including subseries Lecture Notes in Artificial Intelligence and Lecture Notes in Bioinformatics), 7926 LNAI, 908-911. doi: 10.1007/978-3-642-39112-5 143. Descargado de https://www.scopus.com/record/display.uri?eid=2-s2.0$84880011305 \&$ doi $=10.1007 \% 2$ f978-3-642-39112-5$\underline{143 \& \text { origin }=\text { inward\&txGid }=\mathrm{f} 45 \mathrm{~b} 763 \mathrm{~d} 74 \mathrm{~b} 7218 \mathrm{ce} 79425251581 \mathrm{ce} 30}$

Cabada, R. Z., Estrada, M. L. B., Beltran V., J. A., Cibrian R., F., Garcia, C. A. R., \& Perez, Y. H. (2012, julio). Fermat: Merging Affective Tutoring Systems with Learning Social Networks. En 2012 IEEE 12th International Conference on Advanced Learning Technologies (pp. 337-339). Rome: IEEE. doi: 10.1109/ICALT.2012.140. Descargado de https://ieeexplore.ieee.org/document/6268112/ 
Cabada, R. Z., Estrada, M. L. B., Hernández, F. G., \& Bustillos, R. O. (2015). An affective learning environment for Java. En 2015 IEEE 15th International Conference on Advanced Learning Technologies (pp. 350-354). doi: 10.1109/ICALT.2015.53 [GS Search]

Chao, C.-J., Tsai, S.-C., Lee, C.-H., Wang, T.-H., \& Lin, H.-C. (2014). The impact of affective tutoring system and information literacy on elementary school students' cognitive load and learning outcomes. En Workshop Proceedings of the 22nd International Conference on Computers in Education, ICCE 2014 (pp. 847-856). [GS Search]

Chaouachi, M., \& Frasson, C. (2012). Mental workload, engagement and emotions: An exploratory study for intelligent tutoring systems. Lecture Notes in Computer Science (including subseries Lecture Notes in Artificial Intelligence and Lecture Notes in Bioinformatics), 7315 LNCS, 65-71. doi: 10.1007/978-3-642-30950-2 9. Descargado de https://www.scopus.com/inward/record.uri?eid=2-s2.0$\underline{84862503273 \& \text { doi }=10.1007 \% 2 \mathrm{f} 978-3-642-30950 \text { - }}$ 2_9\&partnerID $=40 \& \mathrm{md} 5=476806 \mathrm{f} 9 \mathrm{f} 66064230 \mathrm{a} 9 \mathrm{dfa} 8 \mathrm{ec} 8 \mathrm{fdebf} 2$

Cruces, A. L. L., \& De Arriaga, F. (2000). Reactive agent design for intelligent tutoring systems. Cybernetics \& Systems, 31(1), 1-47. Taylor \& Francis. doi: $\underline{10.1080 / 019697200124900}$ [GS Search]

D’Mello, S., Lehman, B., \& Person, N. (2010). Monitoring affect states during effortful problemsolving activities. International Journal of Artificial Intelligence in Education, 20(4), 361389. doi: 10.3233/JAI-2010-012. Descargado de https://www.scopus.com/inward/record.uri?eid=2-s2.079959316616\&doi $=10.3233 \% 2 \mathrm{fJAI}-2010$ 012\&partnerID $=40 \& m d 5=07 \mathrm{e} 098 \mathrm{c} 320694 \mathrm{bd} 54 \mathrm{e} 934 \mathrm{a} 0704 \mathrm{fa} 3570$

Duffy, M., \& Azevedo, R. (2015). Motivation matters: Interactions between achievement goals and agent scaffolding for self-regulated learning within an intelligent tutoring system. Computers in Human Behavior, 52, 338-348. doi: 10.1016/j.chb.2015.05.041. Descargado de https://www.scopus.com/inward/record.uri?eid=2-s2.0$\underline{84936744787 \& \text { doi }=10.1016 \% 2 \text { fj.chb.2015.05.041\&partnerID }=40 \& m d 5=0 f 6966 \mathrm{~d} 221 \mathrm{c} 0 \mathrm{a} 8 \mathrm{a}}$ $\underline{5993 \mathrm{bf} 4 \mathrm{e} 0595 \mathrm{fcd} 6 \mathrm{~d}}$

Duffy, M. C., Lajoie, S. P., Pekrun, R., \& Lachapelle, K. (2020). Emotions in medical education: Examining the validity of the Medical Emotion Scale (MES) across authentic medical learning environments. Learning and Instruction, 101150. Elsevier. doi: 10.1016/j.learninstruc.2018.07.001 [GS Search]

Ekman, P., \& Keltner, D. (1997). Universal facial expressions of emotion.

Erümit, A. K., \& Çetin, I. (2020, abril). Design framework of adaptive intelligent tutoring systems. Education and Information Technologies. doi: 10.1007/s10639-020-10182-8. Descargado de http://link.springer.com/10.1007/s10639-020-10182-8

Fotopoulou, E., Zafeiropoulos, A., Feidakis, M., Metafas, D., \& Papavassiliou, S. (2020). An interactive recommender system based on reinforcement learning for improving emotional competences in educational groups. In: Kumar V., Troussas C. (eds) Intelligent Tutoring Systems. ITS 2020. Lecture Notes in Computer Science, vol 12149. Springer, Cham. doi: 10.1007/978-3-030-49663-0_29 [GS Search]

Ghaleb, E., Popa, M., Hortal, E., Asteriadis, S., \& Weiss, G. (2019). Towards Affect Recognition through Interactions with Learning Materials. En Proceedings - 17th IEEE International Conference on Machine Learning and Applications, ICMLA 2018 (pp. 372-379). doi: 10.1109/ICMLA.2018.00062. 
$\underline{85062207088 \& \text { doi }=10.1109 \% 2 \text { fICMLA.2018.00062\&partnerID }=40 \& \mathrm{md} 5=5 \mathrm{a} 9 \mathrm{c} 7 \mathrm{eaa} 1 \mathrm{f} 61 \mathrm{~d}}$ e16968deac4bdf8d764

Graesser, A., Forsyth, C., \& Lehman, B. (2017). Two heads may be better than one: Learning from computer agents in conversational trialogues. Teachers College Record, 119(3). [GS $\underline{\text { Search }}$

Graesser, A. C., Wiemer-Hastings, K., Wiemer-Hastings, P., Kreuz, R., Group, T. R., \& others. (1999). AutoTutor: A simulation of a human tutor. Cognitive Systems Research, 1(1), 35-51. Elsevier. doi: 10.1016/S1389-0417(99)00005-4 [GS Search]

Grafsgaard, J., Wiggins, J., Boyer, K., Wiebe, E., \& Lester, J. (2013). Automatically recognizing facial expression: Predicting engagement and frustration. En Proceedings of the $6^{\text {th }}$ International Conference on Educational Data Mining, EDM 2013. [GS Search]

Gudino-Penaloza, F., Mendoza, M. G., Gress, N. H., \& Vargas, J. M. (2009). Intelligent tutorial system for teaching of probability and statistics at high school in Mexico. World Journal on Educational Technology, 1(2). [GS Search]

Han, J., Zhao, W., Jiang, Q., Oubibi, M., \& Hu, X. (2019). Intelligent tutoring system trends 20062018: A literature review. En Proceedings - 2019 8th International Conference of Educational Innovation through Technology, EITT 2019 (pp. 153-159). doi: 10.1109/EITT.2019.00037. Descargado

https://www.scopus.com/inward/record.uri?eid=2-s2.0-

85077071940\&doi $=10.1109 \%$ 2fEITT.2019.00037\&partnerID=40\&md5=10b90e22ac1868d $\underline{\mathrm{b} 4 \mathrm{e} 9 \mathrm{df} 03 \mathrm{e} 27650 \mathrm{a} 3 \mathrm{~b}}$

Harley, J., Poitras, E., Jarrell, A., Duffy, M., \& Lajoie, S. (2016). Comparing virtual and locationbased augmented reality mobile learning: emotions and learning outcomes. Educational Technology Research and Development, 64(3), 359-388. doi: 10.1007/s11423-015-9420-7. Descargado de https://www.scopus.com/inward/record.uri?eid=2-s2.084953393252\&doi $=10.1007 \% 2$ fs $11423-015-9420$ -

7\&partnerID $=40 \& \mathrm{md} 5=8 \mathrm{~d} 7 \mathrm{df5cf9f8d} 583 \mathrm{~b} 9350 \mathrm{~b} 87 \mathrm{a} 479 \mathrm{f} 3 \mathrm{c} 24$

Hull, A., \& du Boulay, B. (2015). Motivational and metacognitive feedback in SQL-Tutor*. Computer Science Education, 25(2), 238-256. doi: 10.1080/08993408.2015.1033143. Descargado de https://www.scopus.com/inward/record.uri?eid=2-s2.0$\underline{84929516090 \& \text { doi }=10.1080 \% 2 \mathrm{f0} 0893408.2015 .1033143 \& \text { partnerID }=40 \& \mathrm{md} 5=7 \mathrm{a} 2206813}$ $15 \mathrm{a} 2311 \mathrm{~d} 0 \mathrm{ca} 80356 \mathrm{cba} 854 \mathrm{e}$

Hussain, M., Alzoubi, O., Calvo, R., \& D’Mello, S. (2011). Affect detection from multichannel physiology during learning sessions with autotutor. Lecture Notes in Computer Science (including subseries Lecture Notes in Artificial Intelligence and Lecture Notes in Bioinformatics), 6738 LNAI, 131-138. doi: 10.1007/978-3-642-21869-9 19. Descargado de https://www.scopus.com/inward/record.uri?eid=2-s2.079959307563\&doi $=10.1007 \% 2 \mathrm{f} 978-3-642-21869-$ 9 19\&partnerID=40\&md5=57561a130deb88a4ac68850c67b7f1 f0

Jimenez, S., Juarez-Ramirez, R., Castillo, V., Licea, G., Ramírez-Noriega, A., \& Inzunza, S. (2018). A feedback system to provide affective support to students. Computer Applications in Engineering Education, 26(3), 473-483. doi: 10.1002/cae.21900. Descargado de https://www.scopus.com/inward/record.uri?eid=2-s2.0-

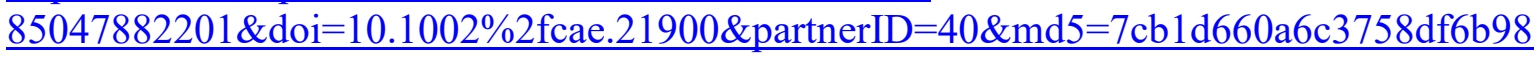
$\underline{2133 \mathrm{~d} 64 \mathrm{bde} 0}$

Joshi, A., Allessio, D., Magee, J., Whitehill, J., Arroyo, I., Woolf, B., ..., \& Betke, M. (2019). Affect-driven learning outcomes prediction in intelligent tutoring systems. En Proceedings - 
14th IEEE International Conference on Automatic Face and Gesture Recognition, FG 2019. doi: 10.1109/FG.2019.8756624. Descargado de https://www.scopus.com/inward/record.uri?eid=2-s2.0-

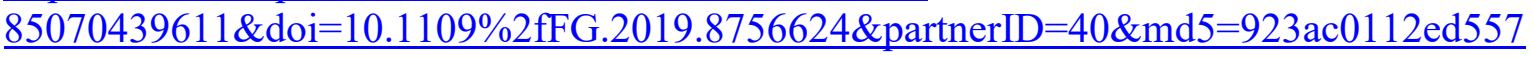
$\underline{8 \mathrm{~d} 8 \mathrm{c} 7359 \mathrm{dc} 810 \mathrm{~b} 2 \mathrm{f} 72}$

Lajoie, S., Zheng, J., Li, S., Jarrell, A., \& Gube, M. (2019). Examining the interplay of affect and self-regulation in the context of clinical reasoning. Learning and Instruction. 72, 101219. doi: 10.1016/j.learninstruc.2019.101219. Descargado

https://www.scopus.com/inward/record.uri?eid=2-s2.0$\underline{85068006597 \& \text { doi }=10.1016 \% 2 \text { fj.learninstruc. } 2019.101219 \& \text { partnerID }=40 \& \mathrm{md} 5=6914 \mathrm{da} 9}$ c10e99c62fa941ff402998450

Lehman, B., D’Mello, S., \& Graesser, A. (2012). Confusion and complex learning during interactions with computer learning environments. Internet and Higher Education, 15(3), 184-194. doi: 10.1016/j.iheduc.2012.01.002. Descargado de https://www.scopus.com/inward/record.uri?eid=2-s2.0-

$\underline{84861734887 \& \text { doi }=10.1016 \% 2 \text { fj.iheduc. } 2012.01 .002 \& \text { partnerID }=40 \& m d 5=e f 9 a 32 a 9 c c 62 b}$ e945112bf1b4cc23db7

Leyzberg, D., Spaulding, S., \& Scassellati, B. (2014). Personalizing robot tutors to individuals' learning differences. En ACM/IEEE International Conference on Human- Robot Interaction (pp. 423-430). doi: 10.1145/2559636.2559671. Descargado de https://www.scopus.com/inward/record.uri?eid=2-s2.0$\underline{84896904456 \& \text { doi }=10.1145 \% 2 \mathrm{f} 2559636.2559671 \& \text { partnerID }=40 \& \mathrm{md} 5=\mathrm{a} 6 \mathrm{f} 5512554 \mathrm{fda} 3 \mathrm{~b}}$ $\underline{1 \mathrm{cc} 00 \mathrm{a} 4732 \mathrm{bb} 27 \mathrm{ddd}}$

Liu, Z., Baker, R., Pataranutaporn, V., \& Ocumpaugh, J. (2013). Sequences of frustration and confusion, and learning. En Proceedings of the 6th International Conference on Educational Data Mining, EDM 2013. [GS Search]

Long, Y., \& Aleven, V. (2017). Educational Game and Intelligent Tutoring System: A Classroom Study and Comparative Design Analysis. ACM Transactions on Computer-Human Interaction, 24(3), 20. doi: 10.1145/3057889. Descargado de https://app.dimensions.ai/details/publication/pub.1085093673

Long, Z., Luo, D., Xu, S., \& Hu, X. (2019). Agents' cognitive vs. Socio-affective support in response to learner's confusion. Lecture Notes in Computer Science (including subseries Lecture Notes in Artificial Intelligence and Lecture Notes in Bioinformatics), 11528 LNCS, 233-235. [GS Search]

Magner, U., Schwonke, R., Renkl, A., Aleven, V., \& Popescu, O. (2010). Pictorial illustrations in intelligent tutoring systems: Do they distract or elicit interest and engagement? En Learning in the Disciplines: ICLS 2010 Conference Proceedings - 9th International Conference of the Learning Sciences (Vol. 1, pp. 1-8). [GS Search]

Mahmoud, M. H. (2019, noviembre). A Survey of Some Interdisciplinary Methods and Tools to Measure Learners' Emotions in Intelligent Tutoring Systems. En 2019 6th International Conference on Advanced Control Circuits and Systems (ACCS) \& 2019 5th International Conference on New Paradigms in Electronics \& information Technology (PEIT) (pp. 1-6). Hurgada, Egypt: IEEE. doi: 10.1109/ACCS-PEIT48329.2019.9062885. Descargado 202008-19, de https://ieeexplore.ieee.org/document/9062885/

Marco-Gimenez, L., Arevalillo-Herraez, M., Ferri, F., Moreno-Picot, S., Boticario, J., Santos, O., ..., \& Ramzan, N. (2016). Affective and behavioral assessment for adaptive intelligent tutoring systems. En CEUR Workshop Proceedings (Vol. 1618). [GS Search] 
Martin, J. M., Ortigosa, A., \& Carro, R. M. (2012). SentBuk: Sentiment analysis for e-learning environments. En 2012 International Symposium on Computers in Education (SIIE) (pp. 16). [GS Search]

Mayer, R. E. (2019). Searching for the role of emotions in e-learning. Learning and Instruction, 70, 101213. doi: 10.1016/j.learninstruc.2019.05.010 [GS Search]

McQuiggan, S., Robison, J., \& Lester, J. (2008). Affective transitions in narrative-centered learning environments. Lecture Notes in Computer Science (including subseries Lecture Notes in Artificial Intelligence and Lecture Notes in Bioinformatics), 5091 LNCS, 490-499. doi: $\quad$ 10.1007/978-3-540-69132-7 52. Descargado de https://www.scopus.com/inward/record.uri?eid=2-s2.070349878682\&doi $=10.1007 \% 2 \mathrm{f} 978-3-540-69132-7-$ 52\&partnerID $=40 \& \mathrm{md} 5=0 \mathrm{ac} 3$ dae1baf2726375379354907b639b

Mohanan, R., Stringfellow, C., \& Gupta, D. (2017, julio). An emotionally intelligent tutoring system. En 2017 Computing Conference (pp. 1099-1107). London: IEEE. doi: 10.1109/SAI.2017.8252228. Descargado http://ieeexplore.ieee.org/document/8252228/

Mousavinasab, E., Zarifsanaiey, N., R. Niakan Kalhori, S., Rakhshan, M., Keikha, L., \& Ghazi Saeedi, M. (2021). Intelligent tutoring systems: a systematic review of characteristics, applications, and evaluation methods. Interactive Learning Environments, 29(1), 142-163. doi: $10.1080 / 10494820.2018 .1558257$ [GS Search]

Muldner, K., Wixon, M., Rai, D., Burleson, W., Woolf, B., \& Arroyo, I. (2015). Exploring the impact of a learning dashboard on student affect. Lecture Notes in Computer Science (including subseries Lecture Notes in Artificial Intelligence and Lecture Notes in Bioinformatics), 9112, 307-317. doi: 10.1007/978-3-319-19773-9 31. Descargado de https://www.scopus.com/inward/record.uri?eid=2-s2.0$\underline{84948987947 \& \text { doi }=10.1007 \% 2 \mathrm{f978}-3-319-19773-}$ 9 31\&partnerID $=40 \& \mathrm{md} 5=649 \mathrm{f} 72 \mathrm{fe} 909 \mathrm{dfa} 44 \mathrm{~cd} 5700 \mathrm{dcbe} 1 \mathrm{e} 9 \mathrm{a} 8 \mathrm{a}$

Muñoz, K., Kevitt, P., Lunney, T., Noguez, J., \& Neri, L. (2010). PlayPhysics: An emotional game learning environment for teaching physics. Lecture Notes in Computer Science (including subseries Lecture Notes in Artificial Intelligence and Lecture Notes in Bioinformatics), 6291 LNAI, 400-411. doi: 10.1007/978-3-642-15280-1_37. Descargado de https://www.scopus.com/inward/record.uri?eid=2-s2.078049392263\&doi $=10.1007 \% 2 \mathrm{f9} 978-3-642-15280-$ 1 37\&partnerID $=40 \& \mathrm{md} 5=\mathrm{f} 66 \mathrm{c} 9 \mathrm{~b} 0 \mathrm{a} 303 \mathrm{ee} 3118 \mathrm{f} 88 \mathrm{e} 048947 \mathrm{c} 24 \mathrm{~b} 5$

Nakamura, J., \& Csikszentmihalyi, M. (2014). The concept of flow. En Flow and the foundations of positive psychology (pp. 239-263). doi: 10.1007/978-94-017-9088-8 16 [GS Search]

Okoli, C., \& Schabram, K. (2010). A Guide to Conducting a Systematic Literature Review of Information Systems Research. SSRN Electronic Journal. doi: 10.2139/ssrn.1954824. Descargado 2020-10-31, de http://www.ssrn.com/abstract=1954824

Ortony, A., Clore, G. L., \& Collins, A. (1988). The cognitive structure of emotions. Cambridge: Cambridge University Press. doi: $\underline{10.1017 / C B O 9780511571299}$

Padron-Rivera, G., Joaquin-Salas, C., Patoni-Nieves, J.-L., \& Bravo-Perez, J.-C. (2018). Patterns in Poor Learning Engagement in Students While They Are Solving Mathematics Exercises in an Affective Tutoring System Related to Frustration. Lecture Notes in Computer Science (including subseries Lecture Notes in Artificial Intelligence and Lecture Notes in Bioinformatics), 10880 LNCS, 169-177. doi: 10.1007/978-3-319-92198-3 17. Descargado de

https://www.scopus.com/inward/record.uri?eid=2-s2.0- 
$\underline{85049325808 \& \text { doi }=10.1007 \% 2 \mathrm{f9} 978-3-319-92198-}$ 3 17\&partnerID $=40 \& \mathrm{md} 5=\mathrm{cb} 4 \mathrm{~b} 0719 \mathrm{~d} 9 \mathrm{bd} 3 \mathrm{f} 1825147 \mathrm{eecb} 1742 \mathrm{~d} 15$

Padrón-Rivera, G., Rebolledo-Mendez, G., Parra, P., \& Huerta-Pacheco, N. (2016). Identification of action units related to affective states in a tutoring system for mathematics. Educational Technology and Society, 19(2), 77-86. [GS Search]

Pardos, Z., Baker, R., San Pedro, M., Gowda, S., \& Gowda, S. (2013). Affective states and state tests: Investigating how affect throughout the school year predicts end of year learning outcomes. En ACM International Conference Proceeding Series (pp. 117-124). doi: $10.1145 / 2460296.2460320$. Descargado

https://www.scopus.com/inward/record.uri?eid=2-s2.0-

$\underline{84876461451 \& \text { doi }=10.1145 \% 2 \mathrm{f} 2460296.2460320 \& \text { partnerID }=40 \& \mathrm{md} 5=43 \mathrm{beba} 0 \mathrm{~d} 43 \mathrm{e} 0343}$ $\underline{405 \mathrm{e} 8 \mathrm{ba} 3286 \mathrm{~d} 208 \mathrm{a} 5}$

Pedroza-Méndez, B. E., Gonzalez-Calleros, J. M., Guerrero-García, J., \& Collazos, C. A. (2019). Continuous Evaluation of the Learning Process of Algebra Through a Semi-Automated Tool. Journal of Information Technology Research, 12(3), 1-20. doi: 10.4018/JITR.2019070101. Descargado de https://app.dimensions.ai/details/publication/pub.1119999110andhttp://pdfs.semanticscholar .org/d6fc/9ad47307c792ab5bb52b1113d9c4c3e9841a.pdf

Pekrun, R. (1992). The impact of emotions on learning and achievement: Towards a theory of cognitive/motivational mediators. Applied Psychology, 41(4), 359-376. Wiley Online Library. doi: 10.1111/j.1464-0597.1992.tb00712.x [GS Search]

Pham, P., \& Wang, J. (2018). Predicting learners' emotions in mobile MOOC learning via a multimodal intelligent tutor. Lecture Notes in Computer Science (including subseries Lecture Notes in Artificial Intelligence and Lecture Notes in Bioinformatics), 10858 LNCS, 150-159. doi: $\quad$ 10.1007/978-3-319-91464-0 15.

https://www.scopus.com/inward/record.uri?eid=2-s2.0$85048327959 \&$ doi $=10.1007 \% 2 \mathrm{f} 978-3-319-91464-$

0 15\&partnerID $=40 \& \mathrm{md} 5=\mathrm{c} 6 \mathrm{c} 14 \mathrm{bbdb} 675 \mathrm{a} 8 \mathrm{f04be} 4685 \mathrm{af9e} 3 \mathrm{a} 90 \mathrm{~d}$

Price, M., Mudrick, N., Taub, M., \& Azevedo, R. (2018). The role of negative emotions and emotion regulation on self-regulated learning with MetaTutor. Lecture Notes in Computer Science (including subseries Lecture Notes in Artificial Intelligence and Lecture Notes in Bioinformatics), 10858 LNCS, 170-179. doi: 10.1007/978-3-319-91464-0_17. Descargado de $\quad$ https://www.scopus.com/inward/record.uri?eid=2-s2.0$\underline{85048331537 \& \text { doi }=10.1007 \% 2 \mathrm{f} 978-3-319-91464-}$

0 17\&partnerID $=40 \& \mathrm{md} 5=8 \mathrm{~d} 9 \mathrm{a} 4 \mathrm{de} 04 \mathrm{dd} 7 \mathrm{bd} 15 \mathrm{dd} 9649 \mathrm{bb} 7 \mathrm{c} 5 \mathrm{a} 9 \mathrm{fae}$

Richey, J., Andres-Bray, J., Mogessie, M., Scruggs, R., Andres, J., Star, J., ..., \& McLaren, B. (2019). More confusion and frustration, better learning: The impact of erroneous examples. Computers and Education, 139, 173-190. doi: 10.1016/j.compedu.2019.05.012. Descargado de https://www.scopus.com/inward/record.uri?eid=2-s2.085067959303\&doi $=10.1016 \% 2$ fj.compedu.2019.05.012\&partnerID $=40 \& \mathrm{md} 5=\mathrm{c} 4 \mathrm{a} 4 \mathrm{db} 9574$ $\underline{0 \mathrm{~d} 52842679 \mathrm{a} 428788 \mathrm{bb} 11 \mathrm{~d}}$

Rodrigo, M., Baker, R., Agapito, J., Nabos, J., Repalam, M., Reyes Jr., S., \& San Pedro, M. (2012). The effects of an interactive software agent on student affective dynamics while using an intelligent tutoring system. IEEE Transactions on Affective Computing, 3(2), 224-236. doi: $\quad 10.1109 / \mathrm{T}-\mathrm{AFFC} .2011 .41 . \quad$ Descargado de https://www.scopus.com/inward/record.uri?eid=2-s2.0-84863971844\&doi=10.1109\%2fTAFFC.2011.41\&partnerID=40\&md5=dd0d85be4fea55af2b2a9a668d11e04f 
Ruiz, S., Urretavizcaya, M., Rodríguez, C., \& Fernández-Castro, I. (2020). Predicting students' outcomes from emotional response in the classroom and attendance. Interactive Learning Environments, 28(1), 107-129. doi: 10.1080/10494820.2018.1528282. Descargado de https://www.scopus.com/inward/record.uri?eid=2-s2.0-

$\underline{85054480130 \& \text { doi }=10.1080 \% 2 \mathrm{f} 10494820.2018 .1528282 \& \text { partnerID }=40 \& \mathrm{md} 5=380 \mathrm{~b} 531 \mathrm{ad}}$ $\underline{850 \mathrm{a} 3 \mathrm{cc} 75 \mathrm{a} 4 \mathrm{~d} 353585 \mathrm{a} 0 \mathrm{a} 9 \mathrm{a}}$

Russell, J. A. (1983). Pancultural aspects of the human conceptual organization of emotions. Journal of personality and social psychology, 45(6), 1281. American Psychological Association. doi: 10.1037/0022-3514.45.6.1281 [GS Search]

Strain, A., D’Mello, S., \& Graesser, A. (2011). Training emotion regulation strategies during computerized learning: A method for improving learner self-regulation. Lecture Notes in Computer Science (including subseries Lecture Notes in Artificial Intelligence and Lecture Notes in Bioinformatics), 6738 LNAI, 617-619. doi: 10.1007/978-3-642-21869-9 119. Descargado de https://www.scopus.com/inward/record.uri?eid=2-s2.0$79959296367 \&$ doi $=10.1007 \% 2 \mathrm{f} 978-3-642-21869-$

9 119\&partnerID $=40 \& \mathrm{md} 5=\mathrm{d} 76336 \mathrm{e} 527 \mathrm{~cd} 6 \mathrm{aca} 820206918620 \mathrm{e} 813$

Taub, M., Sawyer, R., Lester, J., \& Azevedo, R. (2020). The Impact of Contextualized Emotions on Self-Regulated Learning and Scientific Reasoning during Learning with a Game-Based Learning Environment. International Journal of Artificial Intelligence in Education, 30(1), 97-120. doi: 10.1007/s40593-019-00191-1. Descargado de https://www.scopus.com/inward/record.uri?eid=2-s2.0$85076626753 \&$ doi $=10.1007 \% 2$ fs $40593-019-00191-$ $1 \&$ partnerID $=40 \& \mathrm{md} 5=0657 \mathrm{bbcf660 \textrm {dcb } 6 \mathrm { a } 4 2 7 \mathrm { d } 6 \mathrm { f } 4 9 2 8 \mathrm { b } 3 \mathrm { f } 3 \mathrm { b } 6}$

Vail, A., Wiggins, J., Grafsgaard, J., Boyer, K., Wiebe, E., \& Lester, J. (2016). The affective impact of tutor questions: Predicting frustration and engagement. En Proceedings of the 9th International Conference on Educational Data Mining, EDM 2016 (pp. 247-254). doi: 10.1145/1235. Descargado de https://www.scopus.com/inward/record.uri?eid=2-s2.0$\underline{85030214847 \& \text { doi }=10.1145 \% 2 \mathrm{f} 1235 \& \text { partnerID }=40 \& \mathrm{md} 5=3477 \mathrm{e} 19 \mathrm{ea} 241677 \mathrm{be} 335 \mathrm{eeabc} 0}$ $\underline{\mathrm{d} 9 \mathrm{cf} 6 \mathrm{c}}$

VanLehn, K. (2011, octubre). The Relative Effectiveness of Human Tutoring, Intelligent Tutoring Systems, and Other Tutoring Systems. Educational Psychologist, 46(4), 197-221. doi: $10.1080 / 00461520.2011 .611369$. Descargado de http://www.tandfonline.com/doi/abs/10.1080/00461520.2011.611369

Wang, C.-H., \& Lin, H.-C. (2018). Constructing an Affective Tutoring System for Designing Course Learning and Evaluation. Journal of Educational Computing Research, 55(8), 11111128. doi: $\underline{10.1177 / 0735633117699955 .}$ Descargado de https://www.scopus.com/inward/record.uri?eid=2-s2.0-

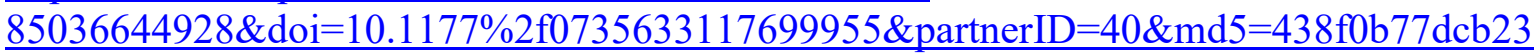
$\underline{\mathrm{f} 4979606 \mathrm{fa} 290033 \mathrm{~d} 03}$

Woolf, B., Arroyo, I., Cooper, D., Burleson, W., \& Muldner, K. (2010). Affective tutors: Automatic detection of and response to student emotion. Studies in Computational Intelligence, 308, 207-227. doi: 10.1007/978-3-642-14363-2_10. Descargado de https://www.scopus.com/inward/record.uri?eid=2-s2.0$77957255983 \&$ doi $=10.1007 \% 2 \mathrm{f} 978-3-642-14363$ 2 10\&partnerID $=40 \& \mathrm{md5}=9630962 \mathrm{a} 1 \mathrm{a} 10 \mathrm{caa} 67 \mathrm{~b} 7 \mathrm{~b} 9 \mathrm{~b} 294195 \mathrm{db} 5 \mathrm{e}$

Xie, H., Chu, H.-C., Hwang, G.-J., \& Wang, C.-C. (2019, octubre). Trends and development in technology-enhanced adaptive/personalized learning: A systematic review of journal publications from 2007 to 2017. Computers \& Education, 140, 103599. doi: 
10.1016/j.compedu.2019.103599. 2020-10-31,

de https://linkinghub.elsevier.com/retrieve/pii/S0360131519301526

Zakharov, K., Mitrovic, A., \& Johnston, L. (2007). Intelligent Tutoring Systems respecting human nature. En Proceedings of NZCSRSC 2007, the 5th New Zealand Computer Science Research Student Conference. [GS Search]

\section{Apéndice 1. Bibliografía del conjunto de documentos obtenido}

Abidi, S. M. R., Zhang, W., Haidery, S. A., Rizvi, S. S., Riaz, R., Ding, H., \& Kwon, S. J. (2020). Educational sustainability through big data assimilation to quantify academic procrastination using ensemble classifiers. Sustainability (Switzerland), 12(15). https://doi.org/10.3390/su12156074

Alabdulhadi, A., \& Faisal, M. (2020). Systematic literature review of STEM self-study related ITSs. Education and Information Technologies. https://doi.org/10.1007/s10639-020-10315-Z

Alhasan, K., Chen, L., \& Chen, F. (2019). An Experimental Study of Learning Behaviour in an ELearning Environment. Proceedings - 20th International Conference on High Performance Computing and Communications, 16th International Conference on Smart City and 4th International Conference on Data Science and Systems, HPCC/SmartCity/DSS 2018, 1398-1403. https://doi.org/10.1109/HPCC/SmartCity/DSS.2018.00231

Al-Omair, O. M., \& Huang, S. (2019). An Emotionally Adaptive Framework for E-Learning Systems. 2nd International Conference on Computer Applications and Information Security, ICCAIS 2019. https://doi.org/10.1109/CAIS.2019.8769547

Alyuz, N. (2016). Shaping the future of education with empathic companions. ERM4CT 2016 - Proceedings of the 2nd Workshop on Emotion Representations and Modelling for Companion Systems, 1-2. https://doi.org/10.1145/3009960.3009964

Andres, J. M. A. L., Paquette, L., Ocumpaugh, J., Jiang, Y., Baker, R. S., Karumbaiah, S., Slater, S., Bosch, N., Munshi, A., Moore, A., \& Biswas, G. (2019). Affect sequences and learning in Betty's brain. ACM International Conference Proceeding Series, $383-390$. https://doi.org/10.1145/3303772.3303807

Argasiński, J. K., \& Węgrzyn, P. (2019). Affective patterns in serious games. Future Generation Computer Systems, 92, 526-538. https://doi.org/10.1016/j.future.2018.06.013

Arguedas, M., Casillas, L., Xhafa, F., Daradoumis, T., Pena, A., \& Caballe, S. (2016). A Fuzzy-Based Approach for Classifying Students' Emotional States in Online Collaborative Work. Proceedings - 2016 10th International Conference on Complex, Intelligent, and Software Intensive Systems, CISIS 2016, 61-68. https://doi.org/10.1109/CISIS.2016.141

Arguel, A., Lockyer, L., Chai, K., Pachman, M., \& Lipp, O. V. (2019). Puzzle-solving activity as an indicator of epistemic confusion. Frontiers in Psychology, 10(JAN). https://doi.org/10.3389/fpsyg.2019.00163

Arroyo, I., Burleson, W., Tai, M., Muldner, K., \& Woolf, B. P. (2013). Gender differences in the use and benefit of advanced learning technologies for mathematics. Journal of Educational Psychology, 105(4), 957-969. https://doi.org/10.1037/a0032748

Arroyo, I., Schultz, S., Wixon, N., Muldner, K., Burleson, W., \& Woolf, B. P. (2016). Addressing affective states with empathy and growth mindset. CEUR Workshop Proceedings, $1618 . \quad$ https://www.scopus.com/inward/record.uri?eid=2-s2.084984638434\&partnerID $=40 \& \mathrm{md} 5=\mathrm{a} 8 \mathrm{f5e} 93 \mathrm{~d} 2856 \mathrm{c} 1 \mathrm{f} 35 \mathrm{f} 9 \mathrm{f5edb} 48 \mathrm{~b} 38557$

Arroyo, I., Wixon, N., Allessio, D., Woolf, B., Muldner, K., \& Burleson, W. (2017). Collaboration improves student interest in online tutoring. Lecture Notes in Computer Science (including subseries Lecture Notes in Artificial Intelligence and Lecture Notes in Bioinformatics), 10331 LNAI, $28-39$. https://doi.org/10.1007/978-3-319-61425-0 3

Arroyo, I., Woolf, B. P., Burelson, W., Muldner, K., Rai, D., \& Tai, M. (2014). A Multimedia Adaptive Tutoring System for Mathematics that Addresses Cognition, Metacognition and Affect. International Journal of Artificial Intelligence in Education, 24(4), 387-426. https://doi.org/10.1007/s40593014-0023-y

Arroyo, I., Woolf, B. P., Cooper, D. G., Burleson, W., \& Muldner, K. (2011). The impact of animated pedagogical agents on girls' and boys' emotions, attitudes, behaviors and learning. Proceedings of the 2011 11th IEEE International Conference on Advanced Learning Technologies, ICALT 2011, 506-510. https://doi.org/10.1109/ICALT.2011.157

Ashwin, T. S., \& Guddeti, R. M. R. (2020). Impact of inquiry interventions on students in e-learning and classroom environments using affective computing framework. User Modeling and User-Adapted Interaction. https://doi.org/10.1007/s11257-019-09254-3

Aslan, S., Okur, E., Alyuz, N., Esme, A. A., \& Baker, R. S. (2019). Human expert labeling process: Valence-arousal labeling for students' affective states. Advances in Intelligent Systems and Computing, 804, 53-61. https://doi.org/10.1007/978-3-319-98872-6 7

Bahreini, K., Nadolski, R., \& Westera, W. (2016). Data Fusion for Real-time Multimodal Emotion Recognition through Webcams and Microphones in ELearning. International Journal of Human-Computer Interaction, 32(5), 415-430. https://doi.org/10.1080/10447318.2016.1159799

Baker, R. S., Hershkovitz, A., Rossi, L. M., Goldstein, A. B., \& Gowda, S. M. (2013). Predicting Robust Learning With the Visual Form of the Moment-byMoment Learning Curve. Journal of the Learning Sciences, 22(4), 639-666. https://doi.org/10.1080/10508406.2013.836653

Baldassarri, S., Hupont, I., Abadía, D., \& Cerezo, E. (2015). Affective-aware tutoring platform for interactive digital television. Multimedia Tools and Applications, 74(9), 3183-3206. https://doi.org/10.1007/s11042-013-1779-Z

Baylor, A. L. (2018). Three research directions for affective learning technologies. Proceedings of International Conference of the Learning Sciences, ICLS, 3(2018-June), 1843-1846.

Behnamnia, N., Kamsin, A., Ismail, M. A. B., \& Hayati, A. (2020). The effective components of creativity in digital game-based learning among young children: A case study. Children and Youth Services Review, 116. https://doi.org/10.1016/i.childyouth.2020.105227

Belland, B. R. (2016). Instructional scaffolding in STEM education: Strategies and efficacy evidence. En Instructional Scaffolding in STEM Education: Strategies and Efficacy Evidence. https://doi.org/10.1007/978-3-319-02565-0

Belpaeme, T., Kennedy, J., Ramachandran, A., Scassellati, B., \& Tanaka, F. (2018). Social robots for education: A review. Science Robotics, 3(21). https://doi.org/10.1126/scirobotics.aat5954

Bernacki, M. L., Aleven, V., \& Nokes-Malach, T. J. (2014). Stability and change in adolescents' task-specific achievement goals and implications for learning mathematics with intelligent tutors. Computers in Human Behavior, 37, 73-80. https://doi.org/10.1016/j.chb.2014.04.009

Bernacki, M. L., Greene, J. A., \& Crompton, H. (2020). Mobile technology, learning, and achievement: Advances in understanding and measuring the role of mobile technology in education. Contemporary Educational Psychology, 60. https://doi.org/10.1016/j.cedpsych.2019.101827

Bernacki, M. L., \& Walkington, C. (2018). The role of situational interest in personalized learning. Journal of Educational Psychology, $110(6)$, 864-881. https://doi.org/10.1037/edu0000250

Bing-You, R., Hayes, V., Varaklis, K., Trowbridge, R., Kemp, H., \& McKelvy, D. (2017). Feedback for Learners in Medical Education: What is Known? A Scoping Review. Academic Medicine, 92(9), 1346-1354. https://doi.org/10.1097/ACM.0000000000001578

Bisra, K., Liu, Q., Nesbit, J. C., Salimi, F., \& Winne, P. H. (2018). Inducing Self-Explanation: A Meta-Analysis. Educational Psychology Review, 30(3), 703-725. https://doi.org/10.1007/s10648-018-9434-x

Bosch, N., \& D'Mello, S. (2013). Programming with your heart on your sleeve: Analyzing the affective states of computer programming students. Lecture Notes in Computer Science (including subseries Lecture Notes in Artificial Intelligence and Lecture Notes in Bioinformatics), 7926 LNAI, $908-911$. https://doi.org/10.1007/978-3-642-39112-5-143 
Bosch, N., D'Mello, S., \& Mills, C. (2013). What emotions do novices experience during their first computer programming learning session? Lecture Notes in Computer Science (including subseries Lecture Notes in Artificial Intelligence and Lecture Notes in Bioinformatics), 7926 LNAI, 11-20. https://doi.org/10.1007/978-3-642-39112-5-2

Botelho, A. F., Baker, R. S., Ocumpaugh, J., \& Heffernan, N. T. (2018). Studying affect dynamics and chronometry using sensor-free detectors. Proceedings of the 11th International Conference on Educational Data Mining, EDM 2018. https://www.scopus.com/inward/record.uri?eid=2-s2.085084016874\&partnerID $=40 \& \mathrm{md} 5=7 \mathrm{ad} 8 \mathrm{ed} 2 \mathrm{~d} 7 \mathrm{~d} 82 \mathrm{bc} 4 \mathrm{~d} 2 \mathrm{~b} 678 \mathrm{e} 7 \mathrm{a} 7307914 \mathrm{f}$

Brom, C., Hannemann, T., Stárková, T., Bromová, E., \& Děchtěrenko, F. (2017). The role of cultural background in the personalization principle: Five experiments with Czech learners. Computers and Education, 112, 37-68. https://doi.org/10.1016/j.compedu.2017.01.001

Cabada, R. Z., Estrada, M. L. B., Beltran V., J. A., Cibrian R., F., Garcia, C. A. R., y Perez, Y. H.(2012, julio). Fermat: Merging Affective Tutoring Systems with Learning Social Networks.En2012 IEEE 12th International Conference on Advanced Learning Technologies(pp.337-339). Rome: IEEE.

Carpenter, D., Emerson, A., Mott, B. W., Saleh, A., Glazewski, K. D., Hmelo-Silver, C. E., \& Lester, J. C. (2020). Detecting off-task behavior from student dialogue in game-based collaborative learning. Lecture Notes in Computer Science (including subseries Lecture Notes in Artificial Intelligence and Lecture Notes in Bioinformatics), 12163 LNAI, 55-66. https://doi.org/10.1007/978-3-030-52237-7 5

Castillo-Manzano, J. I., Castro-Nunõ, M., López-Valpuesta, L., Sanz-Diáz, M. T., \& Yñiguez, R. (2016). Measuring the effect of ARS on academic performance: A global meta-analysis. Computers and Education, 96, 109-121. https://doi.org/10.1016/j.compedu.2016.02.007

Cerezo, R., Esteban, M., Sánchez-Santillán, M., \& Núñez, J. C. (2017). Procrastinating behavior in computer-based learning environments to predict performance: A case study in Moodle. Frontiers in Psychology, 8(AUG). https://doi.org/10.3389/fpsyg.2017.01403

Chao, C.-J., Tsai, S.-C., Lee, C.-H., Wang, T.-H., \& Lin, H.-C. K. (2014). The impact of affective tutoring system and information literacy on elementary school students' cognitive load and learning outcomes. Workshop Proceedings of the 22nd International Conference on Computers in Education, ICCE 2014, 847-856. https://www.scopus.com/inward/record.uri?eid=2-s2.084923974806\&partnerID=40\&md5=090b14105e3536c4ba477d2c0ede7066

Chaouachi, M., \& Frasson, C. (2012). Mental workload, engagement and emotions: An exploratory study for intelligent tutoring systems. Lecture Notes in Computer Science (including subseries Lecture Notes in Artificial Intelligence and Lecture Notes in Bioinformatics), 7315 LNCS, 65-71. https://doi.org/10.1007/978-3-642-30950-2 9

Chaouachi, M., Jraidi, I., \& Frasson, C. (2015). MENTOR: A Physiologically Controlled Tutoring System. Lecture Notes in Computer Science (including subseries Lecture Notes in Artificial Intelligence and Lecture Notes in Bioinformatics), 9146, 56-67. https://doi.org/10.1007/978-3-319-20267-9 5

Chaouachi, M., Jraidi, I., Lajoie, S. P., \& Frasson, C. (2019). Enhancing the learning experience using real-time cognitive evaluation. International Journal of Information and Education Technology, 9(10), 678-688. https://doi.org/10.18178/ijiet.2019.9.10.1287

Charitopoulos, A., Rangoussi, M., \& Koulouriotis, D. (2020). On the Use of Soft Computing Methods in Educational Data Mining and Learning Analytics Research: A Review of Years 2010-2018. International Journal of Artificial Intelligence in Education. https://doi.org/10.1007/s40593-020-00200$\underline{8}$

Chi, M. T. H., Adams, J., Bogusch, E. B., Bruchok, C., Kang, S., Lancaster, M., Levy, R., Li, N., McEldoon, K. L., Stump, G. S., Wylie, R., Xu, D., \& Yaghmourian, D. L. (2018). Translating the ICAP Theory of Cognitive Engagement Into Practice. Cognitive Science, 42(6), $1777-1832$. https://doi.org/10.1111/cogs.12626

Cirett Galán, F., \& Beal, C. R. (2012). EEG estimates of engagement and cognitive workload predict math problem solving outcomes. Lecture Notes in Computer Science (including subseries Lecture Notes in Artificial Intelligence and Lecture Notes in Bioinformatics), 7379 LNCS, $51-62$. https://doi.org/10.1007/978-3-642-31454-4 5

Craig, S. D., \& Schroeder, N. L. (2017). Reconsidering the voice effect when learning from a virtual human. Computers and Education, 114, 193-205. https://doi.org/10.1016/i.compedu.2017.07.003

Cutumisu, M., Chin, D. B., \& Schwartz, D. L. (2019). A digital game-based assessment of middle-school and college students' choices to seek critical feedback and to revise. British Journal of Educational Technology, 50(6), 2977-3003. https://doi.org/10.1111/bjet.12796

Cutumisu, M., \& Schwartz, D. L. (2018). The impact of critical feedback choice on students' revision, performance, learning, and memory. Computers in Human Behavior, 78, 351-367. https://doi.org/10.1016/j.chb.2017.06.029

Dawood, A., Turner, S., \& Perepa, P. (2019). Natural-Spontaneous Affective-Cognitive Dataset for Adult Students with and Without Asperger Syndrome. IEEE Access, 7, 77990-77999. https://doi.org/10.1109/ACCESS.2019.2921914

de Palo, V., Limone, P., Monacis, L., Ceglie, F., \& Sinatra, M. (2018). Enhancing e-learning in old age. Australian Journal of Adult Learning, 58(1), 88109.

DeFalco, J. A., Rowe, J. P., Paquette, L., Georgoulas-Sherry, V., Brawner, K., Mott, B. W., Baker, R. S., \& Lester, J. C. (2018). Detecting and Addressing Frustration in a Serious Game for Military Training. International Journal of Artificial Intelligence in Education, $28(2), 152-193$. https://doi.org/10.1007/s40593-017-0152-1

Dias, R. D., Gupta, A., \& Yule, S. J. (2019). Using Machine Learning to Assess Physician Competence: A Systematic Review. Academic Medicine, 94(3), 427-439. https://doi.org/10.1097/ACM.0000000000002414

D'Mello, S. K., Lehman, B., \& Person, N. (2010). Monitoring affect states during effortful problem solving activities. International Journal of Artificial Intelligence in Education, 20(4), 361-389. https://doi.org/10.3233/JAI-2010-012

D’Mello, S., Person, N., \& Lehman, B. (2009). Antecedent-consequent relationships and cyclical patterns between affective states and problem solving outcomes. Frontiers in Artificial Intelligence and Applications, 200(1), 57-64. https://doi.org/10.3233/978-1-60750-028-5-57

Dowell, N. M. M., Nixon, T. M., \& Graesser, A. C. (2019). Group communication analysis: A computational linguistics approach for detecting sociocognitive roles in multiparty interactions. Behavior Research Methods, 51(3), 1007-1041. https://doi.org/10.3758/s13428-018-1102-z

Duffy, M. C., \& Azevedo, R. (2015). Motivation matters: Interactions between achievement goals and agent scaffolding for self-regulated learning within an intelligent tutoring system. Computers in Human Behavior, 52, 338-348. https://doi.org/10.1016/j.chb.2015.05.041

Ernst, S.-J., Janson, A., Söllner, M., \& Leimeister, J. M. (2016). It's about understanding each other's culture-Improving the outcomes of mobile learning by avoiding culture conflicts. 2016 International Conference on Information Systems, ICIS 2016. https://www.scopus.com/inward/record.uri?eid=2$\underline{\mathrm{s} 2.0-85032106984 \& \text { partnerID }=40 \& \mathrm{md} 5=40 \mathrm{~b} 35 \mathrm{c} 138 \mathrm{~b} 4 \mathrm{c} 3 \mathrm{f} 76 \mathrm{ffd} 52 \mathrm{f} 400 \mathrm{e} 0 \mathrm{da} 34 \mathrm{~d}}$

Evens, M., \& Michael, J. (2005). One-on-one tutoring by humans and computers. En One-on-One Tutoring by Humans and Computers. https://doi.org/10.4324/9781410617071

Felicia, P. (2009). Modelling players' behaviours and learning strategies in video games. Proceedings of the European Conference on Games-based Learning, 2009-January, $120-123$. $84883524230 \&$ partnerID $=40 \& \mathrm{md} 5=8 \mathrm{~d} 1442 \mathrm{f} 6 \mathrm{caa} 9 \mathrm{bb} 0 \mathrm{f} 7760 \mathrm{e} 27 \mathrm{f} 90 \mathrm{a} 2837 \mathrm{f}$

Ghaleb, E., Popa, M., Hortal, E., Asteriadis, S., \& Weiss, G. (2019). Towards Affect Recognition through Interactions with Learning Materials. Proceedings - 17th IEEE International Conference on Machine Learning and Applications, ICMLA 2018, 372-379. https://doi.org/10.1109/ICMLA.2018.00062

Ghergulescu, I., \& Muntean, C. H. (2016). ToTCompute: A Novel EEG-Based TimeOnTask Threshold Computation Mechanism for Engagement Modelling and Monitoring. International Journal of Artificial Intelligence in Education, 26(3), 821-854. https://doi.org/10.1007/s40593-016-0111-2

Ginns, P., Martin, A. J., \& Marsh, H. W. (2013). Designing Instructional Text in a Conversational Style: A Meta-analysis. Educational Psychology Review, 25(4), 445-472. https://doi.org/10.1007/s10648-013-9228-0

Goldberg, B., \& Cannon-Bowers, J. (2015). Feedback source modality effects on training outcomes in a serious game: Pedagogical agents make a difference. Computers in Human Behavior, 52, 1-11. https://doi.org/10.1016/j.chb.2015.05.008

Goldberg, B., Schatz, S., \& Nicholson, D. (2016). A practitioner's guide to personalized instruction. En Advances in Cognitive Ergonomics. https:/www.scopus.com/inward/record.uri?eid=2-s2.0-85051995768\&partnerID=40\&md5=cafc8334087d2b598de7acf1 17d 13da9

Graesser, A. C., Forsyth, C. M., \& Lehman, B. A. (2017). Two heads may be better than one: Learning from computer agents in conversational trialogues. $\begin{array}{llll}\text { Teachers Record, } & \text { College } & \text { https://www.scopus.com/inward/record.uri?eid=2-s2.0- }\end{array}$ 85016646668\&partnerID $=40 \& \mathrm{md} 5=\mathrm{f} 87 \mathrm{bf} 3 \mathrm{e} 47 \mathrm{ef} 05 \mathrm{de} 03 \mathrm{~d} 883 \mathrm{c} 450 \mathrm{a} 2384 \mathrm{~b} 3$ 
Grafsgaard, J. F., Wiggins, J. B., Boyer, K. E., Wiebe, E. N., \& Lester, J. C. (2013a). Automatically recognizing facial expression: Predicting engagement and frustration. Proceedings of the 6th International Conference on Educational Data Mining, EDM 2013. https://www.scopus.com/inward/record.uri?eid=2-s2.0-85084013878\&partnerID=40\&md5=5782784a07d3d3617abde443438805af

Grafsgaard, J. F., Wiggins, J. B., Boyer, K. E., Wiebe, E. N., \& Lester, J. C. (2013b). Embodied affect in tutorial dialogue: Student gesture and posture. Lecture Notes in Computer Science (including subseries Lecture Notes in Artificial Intelligence and Lecture Notes in Bioinformatics), 7926 LNAI, 1-10. https://doi.org/10.1007/978-3-642-39112-5-1

Gudino-Penaloza, F., Mendoza, M. G., Gress, N. H., y Vargas, J. M. (2009). Intelligent tutorial system for teaching of probability and statistics at high school in Mexico.

Guo, Y. R., \& Goh, D. H.-L. (2015). Affect in embodied pedagogical agents: Meta-analytic review. Journal of Educational Computing Research, 53(1), 124-149. https://doi.org/10.1177/0735633115588774

Guy, R. G. (2016). Interactivity and E-learning: The student perspective. En Interactive Learning: Strategies, Technologies and Effectiveness. https://www.scopus.com/inward/record.uri?eid=2-s2.0-85016803236\&partnerID=40\&md5=dcb558a4d1 f0f14c06fbd13ace277d14

Hadjileontiadou, S. J., Dias, S. B., Diniz, J. A., \& Hadjileontiadis, L. J. (2015). Fuzzy logic-based modeling in collaborative and blended learning. En Fuzzy Logic-Based Modeling in Collaborative and Blended Learning. https://doi.org/10.4018/978-1-4666-8705-9

Hainey, T., Soflano, M., \& Connolly, T. M. (2013). A randomized controlled trial to evaluate learning effectiveness using an adaptive serious game to teach SQL at higher education level. En Psychology, Pedagogy, and Assessment in Serious Games. https://doi.org/10.4018/978-1-4666-4773-2.ch013

Halverson, L. R., \& Graham, C. R. (2019). Learner engagement in blended learning environments: A conceptual framework. Online Learning Journal, 23(2), 145-178. https://doi.org/10.24059/olj.v23i2.1481

Han, J., Zhao, W., Jiang, Q., Oubibi, M., \& Hu, X. (2019). Intelligent tutoring system trends 2006-2018: A literature review. Proceedings - 2019 8th International Conference of Educational Innovation through Technology, EITT 2019, 153-159. https://doi.org/10.1109/EITT.2019.00037

Harley, J. M., Poitras, E. G., Jarrell, A., Duffy, M. C., \& Lajoie, S. P. (2016). Comparing virtual and location-based augmented reality mobile learning: Emotions and learning outcomes. Educational Technology Research and Development, 64(3), 359-388. https://doi.org/10.1007/s11423-015-9420-7

Harpstead, E., Elizabeth Richey, J., McLaren, B. M., \& Nguyen, H. (2019). Exploring the subtleties of agency and indirect control in digital learning games. ACM International Conference Proceeding Series, 121-129. https://doi.org/10.1145/3303772.3303797

Hellas, A., Ihantola, P., Petersen, A., Ajanovski, V. V., Gutica, M., Hynninen, T., Knutas, A., Leinonen, J., Messom, C., \& Liao, S. N. (2018). Predicting academic performance: A systematic literature review. Annual Conference on Innovation and Technology in Computer Science Education, ITiCSE, 175-199. https://doi.org/10.1145/3293881.3295783

Hershkovitz, A., Baker, R. S. J. D., Gobert, J., \& Nakama, A. (2012). A data-driven path model of student attributes, affect, and engagement in a computerbased science inquiry microworld. 10th International Conference of the Learning Sciences: The Future of Learning, ICLS 2012 - Proceedings, 1 , 167-174. https://www.scopus.com/inward/record.uri?eid=2-s2.0-84878725309\&partnerID=40\&md5=ba926fc17cb0c7628a048ffaca2df277

Hershkovitz, A., \& Nachmias, R. (2010). Log-based assessment of motivation in online learning. En Handbook of Educational Data Mining. https://doi.org/10.1201/b10274

Hook, K., \& Bunce, D. (2001). Immediate learning in organisational computer training as a function of training intervention affective reaction, and session impact measures. Applied Psychology, 50(3), 436-454. https://doi.org/10.1111/1464-0597.00066

Hull, A., \& du Boulay, B. (2015). Motivational and metacognitive feedback in SQL-Tutor*. Computer Science Education, 25(2), 238-256. https://doi.org/10.1080/08993408.2015.1033143

Hussain, M. S., Alzoubi, O., Calvo, R. A., \& D’Mello, S. K. (2011). Affect detection from multichannel physiology during learning sessions with autotutor. Lecture Notes in Computer Science (including subseries Lecture Notes in Artificial Intelligence and Lecture Notes in Bioinformatics), 6738 LNAI, 131-138. https://doi.org/10.1007/978-3-642-21869-9 19

Intelligent Tutoring Systems-11th International Conference, ITS 2012, Proceedings. (2012). Lecture Notes in Computer Science (including subseries Lecture Notes in Artificial Intelligence and Lecture Notes in Bioinformatics), 7315 LNCS. https://www.scopus.com/inward/record.uri?eid=2-s2.084862489706\&partnerID $=40 \&$ md5 $=159 \mathrm{ef} 3 \mathrm{e} 01 \mathrm{c} 7 \mathrm{bb} 18 \mathrm{c} 48 \mathrm{e} 5 \mathrm{bd} 9802 \mathrm{c} 2 \mathrm{ab} 27$

Jarrell, A., Harley, J. M., Lajoie, S., \& Naismith, L. (2017). Success, failure and emotions: Examining the relationship between performance feedback and emotions in diagnostic reasoning. Educational Technology Research and Development, 65(5), 1263-1284. https://doi.org/10.1007/s11423-017-9521$\underline{6}$

Jiménez, S., Juárez-Ramírez, R., Castillo, V. H., Licea, G., Ramírez-Noriega, A., \& Inzunza, S. (2018). A feedback system to provide affective support to students. Computer Applications in Engineering Education, 26(3), 473-483. https://doi.org/10.1002/cae.21900

Johnson, W. L., \& Lester, J. C. (2016). Face-to-Face Interaction with Pedagogical Agents, Twenty Years Later. International Journal of Artificial Intelligence in Education, 26(1), 25-36. https://doi.org/10.1007/s40593-015-0065-9

Johnson-Glenberg, M. C., Birchfield, D. A., Tolentino, L., \& Koziupa, T. (2014). Collaborative embodied learning in mixed reality motion-capture environments: Two science studies. Journal of Educational Psychology, 106(1), 86-104. https://doi.org/10.1037/a0034008

Joshi, A., Allessio, D., Magee, J., Whitehill, J., Arroyo, I., Woolf, B., Sclaroff, S., \& Betke, M. (2019). Affect-driven learning outcomes prediction in intelligent tutoring systems. Proceedings - 14th IEEE International Conference on Automatic Face and Gesture Recognition, FG 2019. https://doi.org/10.1109/FG.2019.8756624

Karyotis, C., Doctor, F., Iqbal, R., James, A., \& Chang, V. (2016). A fuzzy modelling approach of emotion for affective computing systems. IoTBD 2016 Proceedings of the International Conference on Internet of Things and Big Data, 453-460. https://doi.org/10.5220/0005945604530460

Khan, A., Ahmad, F. H., \& Malik, M. M. (2017). Use of digital game based learning and gamification in secondary school science: The effect on student engagement, learning and gender difference. Education and Information Technologies, 22(6), 2767-2804. https://doi.org/10.1007/s10639-017-96221

Khedher, A. B., Jraidi, I., \& Frasson, C. (2017). Assessing learners' reasoning using eye tracking and a sequence alignment method. Lecture Notes in Computer Science (including subseries Lecture Notes in Artificial Intelligence and Lecture Notes in Bioinformatics), 10362 LNCS, $47-57$. https://doi.org/10.1007/978-3-319-63312-1 5

Kim, H. Y., Kim, B., Lee, J., \& Kim, J. (2017). Hey, wake up: Come along with the artificial learning companion to the e-learner's outcomes high! Conference on Human Factors in Computing Systems - Proceedings, Part F127655, 1763-1770. https://doi.org/10.1145/3027063.3053123

Kim, N. J., Belland, B. R., Lefler, M., Andreasen, L., Walker, A., \& Axelrod, D. (2020). Computer-Based Scaffolding Targeting Individual Versus Groups in Problem-Centered Instruction for STEM Education: Meta-analysis. Educational Psychology Review, 32(2), 415-461. https://doi.org/10.1007/s10648-019-09502-3

Kim, Y., \& Baylor, A. L. (2006). Pedagogical agents as learning companions: The role of agent competency and type of interaction. Educational Technology Research and Development, 54(3), 223-243. https://doi.org/10.1007/s11423-006-8805-Z

Krämer, N. C., \& Bente, G. (2010). Personalizing e-Learning. The social effects of pedagogical agents. Educational Psychology Review, $22(1)$, $71-87$. https://doi.org/10.1007/s10648-010-9123-x

Kron, F. W., Fetters, M. D., Scerbo, M. W., White, C. B., Lypson, M. L., Padilla, M. A., Gliva-McConvey, G. A., Belfore, L. A., West, T., Wallace, A. M., Guetterman, T. C., Schleicher, L. S., Kennedy, R. A., Mangrulkar, R. S., Cleary, J. F., Marsella, S. C., \& Becker, D. M. (2017). Using a computer simulation for teaching communication skills: A blinded multisite mixed methods randomized controlled trial. Patient Education and Counseling, 100(4), 748-759. https://doi.org/10.1016/j.pec.2016.10.024

Kumar, R., \& Rosé, C. P. (2011). Comparing triggering policies for social behaviors. Proceedings of the SIGDIAL 2011 Conference: 12 th Annual Meeting of the Special Interest Group on Discourse and Dialogue, 227-238. https://www.scopus.com/inward/record.uri?eid=2-s2.084863408125\&partnerID=40\&md5=3a1a5ae1af6e3a23a1bab4d9a457b349

Kümmel, E., Moskaliuk, J., Cress, U., \& Kimmerle, J. (2020). Digital learning environments in higher education: A literature review of the role of individual vs. Social settings for measuring learning outcomes. Education Sciences, 10(3). https://doi.org/10.3390/educsci10030078

Lajoie, S. P., Zheng, J., Li, S., Jarrell, A., \& Gube, M. (2019). Examining the interplay of affect and self-regulation in the context of clinical reasoning. Learning and Instruction. https://doi.org/10.1016/j.learninstruc.2019.101219 
Lee, S. Y., Mott, B. W., \& Lester, J. C. (2010). Optimizing story-based learning: An investigation of student narrative profiles. Lecture Notes in Computer Science (including subseries Lecture Notes in Artificial Intelligence and Lecture Notes in Bioinformatics), 6095 LNCS (PART 2), $155-165$. https://doi.org/10.1007/978-3-642-13437-1 16

Lehman, B., D’Mello, S., \& Graesser, A. (2012). Confusion and complex learning during interactions with computer learning environments. Internet and Higher Education, 15(3), 184-194. https://doi.org/10.1016/j.iheduc.2012.01.002

Lehman, B., D'Mello, S., \& Graesser, A. (2013). Who benefits from confusion induction during learning? An individual differences cluster analysis. Lecture Notes in Computer Science (including subseries Lecture Notes in Artificial Intelligence and Lecture Notes in Bioinformatics), 7926 LNAI, 51-60. https://doi.org/10.1007/978-3-642-39112-5-6

Lewis Johnson, W., \& Lester, J. C. (2018). Pedagogical agents: Back to the future. AI Magazine, 39(2), 33-44. https://doi.org/10.1609/aimag.v39i2.2793

Leyzberg, D., Spaulding, S., \& Scassellati, B. (2014). Personalizing robot tutors to individuals' learning differences. ACM/IEEE International Conference on Human-Robot Interaction, 423-430. https://doi.org/10.1145/2559636.2559671

Li, Y.-X., Ma, L.-L., \& Hung, C.-Y. (2018). Application of multi-touch gestures in science education: Interactive digital simulation for improving students' understanding of optical imaging and learning motivation. ICCE 2018 - 26th International Conference on Computers in Education, Workshop Proceedings, $\quad 138-147$ https://www.scopus.com/inward/record.uri?eid=2-s2.085060065589\&partnerID=40\&md5=1fb31e8f97e415228eb9117fa9589a8c

Lin, L., Ginns, P., Wang, T., \& Zhang, P. (2020). Using a pedagogical agent to deliver conversational style instruction: What benefits can you obtain? Computers and Education, 143. https://doi.org/10.1016/j.compedu.2019.103658

Liu, Z., Baker, R. S. J. D., Pataranutaporn, V., \& Ocumpaugh, J. (2013). Sequences of frustration and confusion, and learning. Proceedings of the 6th International Conference on Educational Data Mining, EDM 2013. https://www.scopus.com/inward/record.uri?eid=2-s2.0$85084012674 \&$ partnerID $=40 \& \mathrm{md} 5=62 \mathrm{e} 7 \mathrm{c} 757 \mathrm{da} 502$ defd6c916acca0f 2185

Lohani, M., Poitras, E. G., \& Stokes, C. (2019). A framework for human-technology social systems: The role of inter-personal interactions. En Examining Multiple Intelligences and Digital Technologies for Enhanced Learning Opportunities. https://doi.org/10.4018/978-1-7998-0249-5.ch014

Long, Z., Luo, D., Gao, H., \& Hu, X. (2019). The effects of rejection sensitivity on confusion regulation during learning in multiagent intelligent tutoring system environments. CEUR Workshop Proceedings, 2501, 77-86. https://www.scopus.com/inward/record.uri?eid=2-s2.0$\underline{85075914148 \& \text { partnerID }=40 \& \mathrm{md} 5=\mathrm{c} 9 \mathrm{~d} 102606 \mathrm{cf} 3 \mathrm{ae} 8 \mathrm{c} 13 \mathrm{~d} 4677 \mathrm{f} 58297 \mathrm{~d} 6 \mathrm{f}}$

Long, Z., Luo, D., Xu, S., \& Hu, X. (2019). Agents' cognitive vs. Socio-affective support in response to learner's confusion. Lecture Notes in Computer Science (including subseries Lecture Notes in Artificial Intelligence and Lecture Notes in Bioinformatics), 11528 LNCS, $233-235$.

Magner, U. I. E., Schwonke, R., Aleven, V., Popescu, O., \& Renkl, A. (2014). Triggering situational interest by decorative illustrations both fosters and hinders learning in computer-based learning environments. Learning and Instruction, 29, 141-152. https://doi.org/10.1016/i.learninstruc.2012.07.002

Magner, U., Schwonke, R., Renkl, A., Aleven, V. A. W. M. M., \& Popescu, O. (2010). Pictorial illustrations in intelligent tutoring systems: Do they distract or elicit interest and engagement? Learning in the Disciplines: ICLS 2010 Conference Proceedings - 9th International Conference of the Learning $\begin{array}{lll}\text { Sciences, } & 1, & \text { https://www.scopus.com/inward/record.uri?eid=2-s2.0- }\end{array}$ 84880528102\&partnerID=40\&md5=9d71e6d8d06695e958589273f289497c

Malchanau, A., Petukhova, V., \& Bunt, H. (2018). Towards integration of cognitive models in dialogue management: Designing the virtual negotiation coach application. Dialogue and Discourse, 9(2), 35-79. https://doi.org/10.5087/dad.2018.202

Marco-Gimenez, L., Arevalillo-Herraez, M., Ferri, F. J., Moreno-Picot, S., Boticario, J. G., Santos, O. C., Salmeron-Majadas, S., Saneiro, M., Uria-Rivas, R., Arnau, D., González-Calero, J. A., Ayesh, A., Cabestrero, R., Quirós, P., Arnau-González, P., \& Ramzan, N. (2016). Affective and behavioral assessment for adaptive intelligent tutoring systems. CEUR Workshop Proceedings, 1618. https://www.scopus.com/inward/record.uri?eid=2-s2.084984596830\&partnerID $=40 \& \mathrm{md} 5=\mathrm{d} 2 \mathrm{f} 3 \mathrm{c} 1 \mathrm{~d} 99 \mathrm{e} 93 \mathrm{~d} 6 \mathrm{~cd} 853 \mathrm{e} 629163 \mathrm{bdf} 17 \mathrm{e}$

Martha, A. S. D., \& Santoso, H. B. (2019). The design and impact of the pedagogical agent: A systematic literature review. Journal of Educators Online, 16(1). https://doi.org/10.9743/jeo.2019.16.1.8

Martha, A. S. D., \& Santoso, H. B. (2018). Investigation of motivation theory on pedagogical agents design in the online learning environment. $A C M$ International Conference Proceeding Series, 217-222. https://doi.org/10.1145/3290511.3290530

McQuiggan, S. W., Robison, J. L., \& Lester, J. C. (2008). Affective transitions in narrative-centered learning environments. Lecture Notes in Computer Science (including subseries Lecture Notes in Artificial Intelligence and Lecture Notes in Bioinformatics), 5091 LNCS, $490-499$. https://doi.org/10.1007/978-3-540-69132-7-52

McQuiggan, S. W., Robison, J. L., \& Lester, J. C. (2010). Affective transitions in narrative-centered learning environments. Educational Technology and Society, 13(1), 40-53.

McQuiggan, S. W., Robison, J. L., Phillips, R., \& Lester, J. C. (2008). Modeling parallel and reactive empathy in virtual agents: An inductive approach. Proceedings of the International Joint Conference on Autonomous Agents and Multiagent Systems, AAMAS, 1, $166-173$. https://www.scopus.com/inward/record.uri?eid=2-s2.0-84899918763\&partnerID=40\&md5=1b50c94e34c9f1c72b98ce08fdea3ed1

Mikheeva, M., Schneider, S., Beege, M., \& Rey, G. D. (2019). Boundary conditions of the politeness effect in online mathematical learning. Computers in Human Behavior, 92, 419-427. https://doi.org/10.1016/j.chb.2018.11.028

Moreno-Marcos, P. M., Martínez de la Torre, D., González Castro, G., Muñoz-Merino, P. J., \& Delgado Kloos, C. (2020). Should we consider efficiency and constancy for adaptation in intelligent tutoring systems? Lecture Notes in Computer Science (including subseries Lecture Notes in Artificial Intelligence and Lecture Notes in Bioinformatics), 12149 LNCS, 237-247. https://doi.org/10.1007/978-3-030-49663-0 28

Muis, K. R., Ranellucci, J., Trevors, G., \& Duffy, M. C. (2015). The effects of technology-mediated immediate feedback on kindergarten students' attitudes, emotions, engagement and learning outcomes during literacy skills development. Learning and Instruction, 38 , 1-13. https://doi.org/10.1016/j.learninstruc.2015.02.001

Muldner, K., \& Conati, C. (2011). A decision-theoretic tutor for analogical problem solving. En Decision Theory Models for Applications in Artificial Intelligence: Concepts and Solutions. https://doi.org/10.4018/978-1-60960-165-2.ch010

Muldner, K., Wixon, M., Rai, D., Burleson, W., Woolf, B., \& Arroyo, I. (2015). Exploring the impact of a learning dashboard on student affect. Lecture Notes in Computer Science (including subseries Lecture Notes in Artificial Intelligence and Lecture Notes in Bioinformatics), 9112, $307-317$. https://doi.org/10.1007/978-3-319-19773-9 31

Muñoz, K., Kevitt, P. M., Lunney, T., Noguez, J., \& Neri, L. (2010). PlayPhysics: An emotional game learning environment for teaching physics. Lecture Notes in Computer Science (including subseries Lecture Notes in Artificial Intelligence and Lecture Notes in Bioinformatics), 6291 LNAI, $400-411$. https://doi.org/10.1007/978-3-642-15280-1 37

Muñoz, K., Kevitt, P. M., Lunney, T., Noguez, J., \& Neri, L. (2011). An emotional student model for game-play adaptation. Entertainment Computing, 2(2), 133-141. https://doi.org/10.1016/j.entcom.2010.12.006

Muñoz, K., Noguez, J., McKevitt, P., Lunney, T., \& Neri, L. (2010). Work in progress-Towards an emotional learning model for intelligent gaming. Proceedings - Frontiers in Education Conference, FIE, T3G1-T3G2. https://doi.org/10.1109/FIE.2010.5673225

Narciss, S., Sosnovsky, S., Schnaubert, L., Andrès, E., Eichelmann, A., Goguadze, G., \& Melis, E. (2014). Exploring feedback and student characteristics relevant for personalizing feedback strategies. Computers and Education, 71, 56-76. https://doi.org/10.1016/j.compedu.2013.09.011

Nawaz, S., Srivastava, N., Yu, J. H., Baker, R. S., Kennedy, G., \& Bailey, J. (2020). Analysis of task difficulty sequences in a simulation-based POE environment. Lecture Notes in Computer Science (including subseries Lecture Notes in Artificial Intelligence and Lecture Notes in Bioinformatics), 12163 LNAI, 423-436. https://doi.org/10.1007/978-3-030-52237-7 34

Noroozi, O., Dehghanzadeh, H., \& Talaee, E. (2020). A systematic review on the impacts of game-based learning on argumentation skills. Entertainment Computing, 35. https://doi.org/10.1016/j.entcom.2020.100369

Ozcelik, E., Cagiltay, N. E., \& Ozcelik, N. S. (2013). The effect of uncertainty on learning in game-like environments. Computers and Education, 67, 12-20. https://doi.org/10.1016/j.compedu.2013.02.009 
Padron-Rivera, G., Joaquin-Salas, C., Patoni-Nieves, J.-L., \& Bravo-Perez, J.-C. (2018). Patterns in Poor Learning Engagement in Students While They Are Solving Mathematics Exercises in an Affective Tutoring System Related to Frustration. Lecture Notes in Computer Science (including subseries Lecture Notes in Artificial Intelligence and Lecture Notes in Bioinformatics), 10880 LNCS, 169-177. https://doi.org/10.1007/978-3-319-92198-3 17

Paquette, L., Baker, R. S. J. D., Sao Pedro, M. A., Gobert, J. D., Rossi, L., Nakama, A., \& Kauffman-Rogoff, Z. (2014). Sensor-free affect detection for a simulation-based science inquiry learning environment. Lecture Notes in Computer Science (including subseries Lecture Notes in Artificial Intelligence and Lecture Notes in Bioinformatics), 8474 LNCS, 1-10. https://doi.org/10.1007/978-3-319-07221-0 1

Pardos, Z. A., Baker, R. S. J. D., San Pedro, M. O. C. Z., Gowda, S. M., \& Gowda, S. M. (2013). Affective states and state tests: Investigating how affect throughout the school year predicts end of year learning outcomes. ACM International Conference Proceeding Series, 117-124. https://doi.org/10.1145/2460296.2460320

Parkes, J., \& Zimmaro, D. (2016). Learning and assessing with multiple-choice questions in college classrooms. En Learning and Assessing with MultipleChoice Questions in College Classrooms. https://doi.org/10.4324/9781315727769

Pedroza-Méndez, B. E., Gonzalez-Calleros, J. M., Guerrero-García, J., y Collazos, C. A. (2019). Continuous Evaluation of the Learning Process of Algebra Through aSemi-Automated Tool.Journal of Information Technology Research,12(3), 1-20.

Pham, P., \& Wang, J. (2018). Predicting learners' emotions in mobile MOOC learning via a multimodal intelligent tutor. Lecture Notes in Computer Science (including subseries Lecture Notes in Artificial Intelligence and Lecture Notes in Bioinformatics), 10858 LNCS, $150-159$. https://doi.org/10.1007/978-3-319-91464-0 15

Price, M. J., Mudrick, N. V., Taub, M., \& Azevedo, R. (2018). The role of negative emotions and emotion regulation on self-regulated learning with MetaTutor. Lecture Notes in Computer Science (including subseries Lecture Notes in Artificial Intelligence and Lecture Notes in Bioinformatics), 10858 LNCS, 170-179. https://doi.org/10.1007/978-3-319-91464-0 17

Psycharis, S. I. (2009). Physics and cognitive-emotional-metacognitive variables: Learning performance in the environment of CTAT. En Handbook of Research on Synthetic Emotions and Sociable Robotics: New Applications in Affective Computing and Artificial Intelligence. https://doi.org/10.4018/978-1-60566-354-8.ch019

Pynadath, D. V., Wang, N., \& Yang, R. (2018). Simulating collaborative learning through decision-theoretic agents. CEUR Workshop Proceedings, 2153, 36-47. https://www.scopus.com/inward/record.uri?eid=2-s2.0-85054937409\&partnerID=40\&md5=31fd024547485dc9270fe9c7c63d4f3f

Ramachandran, A., Huang, C.-M., \& Scassellati, B. (2019). Toward effective robot-child tutoring: Internal motivation, behavioral intervention, and learning outcomes. ACM Transactions on Interactive Intelligent Systems, 9(1). https://doi.org/10.1145/3213768

Reber, R., Hetland, H., Chen, W., Norman, E., \& Kobbeltvedt, T. (2009). Effects of example choice on interest, control, and learning. Journal of the Learning Sciences, 18(4), 509-548. https://doi.org/10.1080/10508400903191896

Richey, J. E., Andres-Bray, J. M. L., Mogessie, M., Scruggs, R., Andres, J. M. A. L., Star, J. R., Baker, R. S., \& McLaren, B. M. (2019). More confusion and frustration, better learning: The impact of erroneous examples. Computers and Education, 139, 173-190. https://doi.org/10.1016/j.compedu.2019.05.012

Riemer, V., \& Schrader, C. (2020). Playing to learn or to win? The role of students' competition preference on self-monitoring and learning outcome when learning with a serious game. Interactive Learning Environments. https://doi.org/10.1080/10494820.2020.1752741

Rittle-Johnson, B., \& Loehr, A. M. (2017). Eliciting explanations: Constraints on when self-explanation aids learning. Psychonomic Bulletin and Review, 24(5), 1501-1510. https://doi.org/10.3758/s13423-016-1079-5

Robison, J. L., McQuiggan, S. W., \& Lester, J. C. (2009). Modeling task-based vs. Affect-based feedback behavior in pedagogical agents: An inductive approach. Frontiers in Artificial Intelligence and Applications, 200(1), 25-32. https://doi.org/10.3233/978-1-60750-028-5-25

Robison, J., McQuiggan, S., \& Lester, J. (2010). Developing empirically based student personality profiles for affective feedback models. Lecture Notes in Computer Science (including subseries Lecture Notes in Artificial Intelligence and Lecture Notes in Bioinformatics), 6094 LNCS (PART 1), 285295. https://doi.org/10.1007/978-3-642-13388-6 33

Rodrigo, M. M. T., Baker, R. S. J. D., Agapito, J., Nabos, J., Repalam, Ma. C., Reyes Jr., S. S., \& San Pedro, M. O. C. Z. (2012). The effects of an interactive software agent on student affective dynamics while using an intelligent tutoring system. IEEE Transactions on Affective Computing, 3(2), $224-236$. https://doi.org/10.1109/T-AFFC.2011.41

Rodrigues, J. J. P. C., João, P. F. N., \& de la Torre Díez, I. (2013). Recent advances in intelligent tutoring systems: A case study. En Handbook of Research on Teaching and Learning in K-20 Education. https://doi.org/10.4018/978-1-4666-4249-2.ch037

Rodriguez, P., Ortigosa, A., \& Carro, R. M. (2012). Extracting emotions from texts in E-learning Environments. Proceedings - 2012 6th International Conference on Complex, Intelligent, and Software Intensive Systems, CISIS 2012, 887-892. https://doi.org/10.1109/CISIS.2012.192

Ruiz, S., Urretavizcaya, M., Rodríguez, C., \& Fernández-Castro, I. (2020). Predicting students' outcomes from emotional response in the classroom and attendance. Interactive Learning Environments, 28(1), 107-129. https://doi.org/10.1080/10494820.2018.1528282

Sabourin, J., Mott, B., \& Lester, J. (2013). Utilizing dynamic Bayes nets to improve early prediction models of self-regulated learning. Lecture Notes in Computer Science (including subseries Lecture Notes in Artificial Intelligence and Lecture Notes in Bioinformatics), 7899 LNCS, $228-241$. https://doi.org/10.1007/978-3-642-38844-6 19

San Pedro, M. O. Z., Baker, R. S., \& Heffernan, N. T. (2017). An Integrated Look at Middle School Engagement and Learning in Digital Environments as Precursors to College Attendance. Technology, Knowledge and Learning, 22(3), 243-270. https://doi.org/10.1007/s10758-017-9318-Z

Sanchez, R. P., Bartel, C. M., Brown, E., \& Derosier, M. (2014). The acceptability and efficacy of an intelligent social tutoring system. Computers and Education, 78, 321-332. https://doi.org/10.1016/j.compedu.2014.06.013

Santos, O. C., \& Corbi, A. (2019). Can Aikido Help with the Comprehension of Physics? A First Step towards the Design of Intelligent Psychomotor Systems for STEAM Kinesthetic Learning Scenarios. IEEE Access, 7, 176458-176469. https://doi.org/10.1109/ACCESS.2019.2957947

Santos, P. B., Bhowmik, C. V., \& Gurevych, I. (2020). Avoiding bias in students' intrinsic motivation detection. Lecture Notes in Computer Science (including subseries Lecture Notes in Artificial Intelligence and Lecture Notes in Bioinformatics), 12149 LNCS, 89-94. https://doi.org/10.1007/9783-030-49663-0 12

Scheiter, K., Schubert, $\bar{C}$., Schüler, A., Schmidt, H., Zimmermann, G., Wassermann, B., Krebs, M.-C., \& Eder, T. (2019). Adaptive multimedia: Using gazecontingent instructional guidance to provide personalized processing support. Computers and Education, 139 , $31-47$. https://doi.org/10.1016/j.compedu.2019.05.005

Schrader, C., \& Bastiaens, T. J. (2012). Educational computer games and learning: The relationship between design, cognitive load emotions and outcomes. Journal of Interactive Learning Research, 23(3), 251-271.

Schroeder, N. L., Romine, W. L., \& Craig, S. D. (2017). Measuring pedagogical agent persona and the influence of agent persona on learning. Computers and Education, 109, 176-186. https://doi.org/10.1016/j.compedu.2017.02.015

Schroeder, N. L., Yang, F., Banerjee, T., Romine, W. L., \& Craig, S. D. (2018). The influence of learners' perceptions of virtual humans on learning transfer. Computers and Education, 126, 170-182. https://doi.org/10.1016/j.compedu.2018.07.005

Schumacher, C., \& Ifenthaler, D. (2018). The importance of students' motivational dispositions for designing learning analytics. Journal of Computing in Higher Education, 30(3), 599-619. https://doi.org/10.1007/s12528-018-9188-y

Sedrakyan, G., Leony, D., Muñoz-Merino, P. J., Kloos, C. D., \& Verbert, K. (2017). Evaluating student-facing learning dashboards of affective states. Lecture Notes in Computer Science (including subseries Lecture Notes in Artificial Intelligence and Lecture Notes in Bioinformatics), 10474 LNCS, 224237. https://doi.org/10.1007/978-3-319-66610-5 17

Shangguan, C., Wang, Z., Gong, S., Guo, Y., \& Xu, S. (2020). More Attractive or More Interactive? The Effects of Multi-Leveled Emotional Design on Middle School Students' Multimedia Learning. Frontiers in Psychology, 10. https://doi.org/10.3389/fpsyg.2019.03065

Sharma, K., \& Giannakos, M. (2020). Multimodal data capabilities for learning: What can multimodal data tell us about learning? British Journal of Educational Technology, 51(5), 1450-1484. https://doi.org/10.1111/bjet.12993

Sharma, K., Papamitsiou, Z., \& Giannakos, M. (2019). Building pipelines for educational data using AI and multimodal analytics: A "grey-box" approach. British Journal of Educational Technology, 50(6), 3004-3031. https://doi.org/10.1111/bjet.12854 
Sharma, K., Papamitsiou, Z., Olsen, J. K., \& Giannakos, M. (2020). Predicting learners' effortful behavior in adaptive assessment using multimodal data. ACM International Conference Proceeding Series, 480-489. https://doi.org/10.1145/3375462.3375498

Shute, V. J., D’Mello, S., Baker, R., Cho, K., Bosch, N., Ocumpaugh, J., Ventura, M., \& Almeda, V. (2015). Modeling how incoming knowledge, persistence, affective states, and in-game progress influence student learning from an educational game. Computers and Education, 86, $224-235$. https://doi.org/10.1016/i.compedu.2015.08.001

Soflano, M., Connolly, T. M., \& Hainey, T. (2015). An application of adaptive games-based learning based on learning style to teach SQL. Computers and Education, 86, 192-211. https://doi.org/10.1016/i.compedu.2015.03.015

Soflano, S., Connolly, T., \& Hainey, T. (2013). An application of adaptive games-based learning based on learning style to teach SQL. 7th European Conference on Games Based Learning, ECGBL 2013, 2, 531-538. https://www.scopus.com/inward/record.uri?eid=2-s2.084893836964\&partnerID $=40 \&$ md5 $=$ ee3e323e3739c2d1 feafebc842877ceb

Sottilare, R. A., Shawn Burke, C., Salas, E., Sinatra, A. M., Johnston, J. H., \& Gilbert, S. B. (2018). Designing Adaptive Instruction for Teams: A MetaAnalysis. International Journal of Artificial Intelligence in Education, 28(2), 225-264. https://doi.org/10.1007/s40593-017-0146-Z

Spann, C. A., Shute, V. J., Rahimi, S., \& D'Mello, S. K. (2019). The productive role of cognitive reappraisal in regulating affect during game-based learning. Computers in Human Behavior, 100, 358-369. https://doi.org/10.1016/i.chb.2019.03.002

Stephan, A. (2015). Empathy for Artificial Agents. International Journal of Social Robotics, 7(1), 111-116. https://doi.org/10.1007/s12369-014-0260-0

Strain, A. C., Azevedo, R., \& D’Mello, S. K. (2013). Using a false biofeedback methodology to explore relationships between learners' affect, metacognition, and performance. Contemporary Educational Psychology, 38(1), 22-39. https://doi.org/10.1016/j.cedpsych.2012.08.001

Strain, A. C., D'Mello, S. K., \& Graesser, A. C. (2011). Training emotion regulation strategies during computerized learning: A method for improving learner self-regulation. Lecture Notes in Computer Science (including subseries Lecture Notes in Artificial Intelligence and Lecture Notes in Bioinformatics), 6738 LNAI, 617-619. https://doi.org/10.1007/978-3-642-21869-9 119

Syed, M., Chetlur, M., Afzal, S., Ambrose, G. A., \& Chawla, N. V. (2019). Implicit and explicit emotions in MOOCs. EDM 2019 - Proceedings of the 12th International Conference on Educational Data Mining, 432-437. https://www.scopus.com/inward/record.uri?eid=2-s2.085085981722\&partnerID $=40 \& m d 5=a 026191043 \mathrm{~d} 345339 \mathrm{a} 3 \mathrm{c} 9 \mathrm{~d} 25 \mathrm{dddd} 4 \mathrm{fe} 3$

Taub, M., Mudrick, N. V., Azevedo, R., Millar, G. C., Rowe, J., \& Lester, J. (2017). Using multi-channel data with multi-level modeling to assess in-game performance during gameplay with CRYSTAL ISLAND. Computers in Human Behavior, 76, 641-655. https://doi.org/10.1016/j.chb.2017.01.038

Taub, M., Sawyer, R., Lester, J., \& Azevedo, R. (2020). The Impact of Contextualized Emotions on Self-Regulated Learning and Scientific Reasoning during Learning with a Game-Based Learning Environment. International Journal of Artificial Intelligence in Education, 30(1), 97-120. https://doi.org/10.1007/s40593-019-00191-1

Taub, M., Sawyer, R., Smith, A., Rowe, J., Azevedo, R., \& Lester, J. (2020). The agency effect: The impact of student agency on learning, emotions, and problem-solving behaviors in a game-based learning environment. Computers and Education, 147. https://doi.org/10.1016/j.compedu.2019.103781

Ting, C.-Y., Sam, Y.-C., \& Wong, C.-O. (2013). Model of conceptual change for INQPRO: A Bayesian Network approach. Computers and Education, 65, 77-91. https://doi.org/10.1016/j.compedu.2013.01.013

Tong, R., Wang, S., McBride, E., Kelly, H., \& Cui, W. (2020). Data, Mark of a New Era. Lecture Notes in Educational Technology, 17-35. https://doi.org/10.1007/978-981-15-4526-9 2

Trifa, A., Hedhili, A., \& Chaari, W. L. (2019). Knowledge tracing with an intelligent agent, in an e-learning platform. Education and Information Technologies, 24(1), 711-741. https://doi.org/10.1007/s10639-018-9792-5

Tumenayu, O. O., \& Shabalina, O. (2013). Digital educational games: Adopting pedagogical agent to infer leaner's motivation and emotional state. 7th European Conference on Games Based Learning, ECGBL 2013, 2, 546-552. https://www.scopus.com/inward/record.uri?eid=2-s2.084893837693\&partnerID $=40 \& m d 5=\mathrm{da} 3 \mathrm{cb} 9 \mathrm{aecca} 0 \mathrm{~d} 3606 \mathrm{ce} 728385 \mathrm{a} 764 \mathrm{dd} 8$

Um, E. R., Plass, J. L., Hayward, E. O., \& Homer, B. D. (2012). Emotional Design in Multimedia Learning. Journal of Educational Psychology, 104(2), 485-498. https://doi.org/10.1037/a0026609

Vaculíková, J. A. (2018). Measuring self-regulated learning and online learning events to predict student academic performance. Studia Paedagogica, 23(4), 91-118. https://doi.org/10.5817/SP2018-4-5

Vail, A. K., Grafsgaard, J. F., Wiggins, J. B., Lester, J. C., \& Boyer, K. E. (2014). Predicting learning and engagement in tutorial dialogue: A personalitybased model. ICMI 2014 - Proceedings of the 2014 International Conference on Multimodal Interaction, $255-262$. https://doi.org/10.1145/2663204.2663276

Vail, A. K., Wiggins, J. B., Grafsgaard, J. F., Boyer, K. E., Wiebe, E. N., \& Lester, J. C. (2016). The affective impact of tutor questions: Predicting frustration and engagement. Proceedings of the 9th International Conference on Educational Data Mining, EDM 2016, 247-254. https://doi.org/10.1145/1235

Van Campenhout, R. (2020). Supporting metacognitive learning strategies through an adaptive application. Lecture Notes in Computer Science (including subseries Lecture Notes in Artificial Intelligence and Lecture Notes in Bioinformatics), 12214 LNCS, 218-227. https://doi.org/10.1007/978-3-03050788-6 16

Veletsianos, G., \& Russell, G. S. (2014). Pedagogical agents. En Handbook of Research on Educational Communications and Technology: Fourth Edition. https://doi.org/10.1007/978-1-4614-3185-5 61

Veliyath, N., De, P., Allen, A. A., Hodges, C. B., \& Mitra, A. (2019). Modeling students' attention in the classroom using eye trackers. ACMSE 2019 Proceedings of the 2019 ACM Southeast Conference, 2-9. https://doi.org/10.1145/3299815.3314424

Wang, C.-H., \& Lin, H.-C. K. (2018). Constructing an Affective Tutoring System for Designing Course Learning and Evaluation. Journal of Educational Computing Research, 55(8), 1111-1128. https://doi.org/10.1177/0735633117699955

Wang, N., Johnson, W. L., Mayer, R. E., Rizzo, P., Shaw, E., \& Collins, H. (2008). The politeness effect: Pedagogical agents and learning outcomes. International Journal of Human Computer Studies, 66(2), 98-112. https://doi.org/10.1016/j.ijhcs.2007.09.003

Wang, Y., \& Sperling, R. A. (2020). Characteristics of Effective Self-Regulated Learning Interventions in Mathematics Classrooms: A Systematic Review. Frontiers in Education, 5. https://doi.org/10.3389/feduc.2020.00058

Wixon, M., Baker, R. S. J. D., Gobert, J. D., Ocumpaugh, J., \& Bachmann, M. (2012). WTF? Detecting students who are conducting inquiry without thinking fastidiously. Lecture Notes in Computer Science (including subseries Lecture Notes in Artificial Intelligence and Lecture Notes in Bioinformatics), 7379 LNCS, 286-296. https://doi.org/10.1007/978-3-642-31454-4 24

Woolf, B. P., Arroyo, I., Cooper, D., Burleson, W., \& Muldner, K. (2010). Affective tutors: Automatic detection of and response to student emotion. Studies in Computational Intelligence, 308, 207-227. https://doi.org/10.1007/978-3-642-14363-2 10

Woolf, B. P., Arroyo, I., Muldner, K., Burleson, W., Cooper, D. G., Dolan, R., \& Christopherson, R. M. (2010). The effect of motivational learning companions on low achieving students and students with disabilities. Lecture Notes in Computer Science (including subseries Lecture Notes in Artificial Intelligence and Lecture Notes in Bioinformatics), 6094 LNCS (PART 1), 327-337. https://doi.org/10.1007/978-3-642-13388-6 37

Wortha, F., Azevedo, R., Taub, M., \& Narciss, S. (2019). Multiple Negative Emotions During Learning With Digital Learning Environments - Evidence on Their Detrimental Effect on Learning From Two Methodological Approaches. Frontiers in Psychology, 10. https://doi.org/10.3389/fpsyg.2019.02678

Xu, T., Zhou, Y., Wang, Z., \& Peng, Y. (2018). Learning Emotions EEG-based Recognition and Brain Activity: A Survey Study on BCI for Intelligent Tutoring System. Procedia Computer Science, 130, 376-382. https://doi.org/10.1016/j.procs.2018.04.056

Yuce, A., Abubakar, A. M., \& Ilkan, M. (2019). Intelligent tutoring systems and learning performance: Applying task-technology fit and IS success model. Online Information Review, 43(4), 600-616. https://doi.org/10.1108/OIR-11-2017-0340

Yun, H., Fortenbacher, A., \& Pinkwart, N. (2017). Improving a mobile learning companion for self-regulated learning using sensors. CSEDU 2017 Proceedings of the 9th International Conference on Computer Supported Education, 1, 531-536. https://doi.org/10.5220/0006375405310536

Zakharov, K., Mitrovic, A., \& Johnston, L. (2007). Intelligent Tutoring Systems respecting human nature. Proceedings of NZCSRSC 2007, the 5th New Zealand Computer Science Research Student Conference. https://www.scopus.com/inward/record.uri?eid=2-s2.0$\underline{84880126235 \& \text { partnerID }=40 \& \mathrm{md} 5=27363 \mathrm{fbbc} 3 \text { bad } 8 \text { bdaed } 825 \mathrm{e} 2 \mathrm{e} 4467891}$ 